\title{
Nanosuspension: An Emerging Trend for Bioavailability Enhancement of Etodolac
}

\author{
Samar A. Afifi, ${ }^{1,2}$ Maha A. Hassan, ${ }^{1,3}$ Ali S. Abdelhameed, ${ }^{4}$ and Kadria A. Elkhodairy ${ }^{1,5}$ \\ ${ }^{1}$ Department of Pharmaceutics, College of Pharmacy, King Saud University, P.O. Box 22452, Riyadh 11495, Saudi Arabia \\ ${ }^{2}$ Department of Pharmaceutics, National Organization for Drug Control and Research, P.O. Box 35521, Giza, Egypt \\ ${ }^{3}$ Department of Pharmaceutics, Faculty of Pharmacy, Assiut University, P.O. Box 71515, Assiut, Egypt \\ ${ }^{4}$ Department of Pharmaceutical Chemistry, College of Pharmacy, King Saud University, P.O. Box 2457, Riyadh 11451, Saudi Arabia \\ ${ }^{5}$ Department of Industrial Pharmacy, Faculty of Pharmacy, Alexandria University, P.O. Box 21500, Alexandria, Egypt
}

Correspondence should be addressed to Kadria A. Elkhodairy; elkhodairy53@yahoo.com

Received 5 October 2014; Accepted 13 January 2015

Academic Editor: Alenka Vesel

Copyright (C) 2015 Samar A. Afifi et al. This is an open access article distributed under the Creative Commons Attribution License, which permits unrestricted use, distribution, and reproduction in any medium, provided the original work is properly cited.

\begin{abstract}
Etodolac (ET) (poorly soluble drug) nanosuspensions were prepared by both $\mathrm{pH}$ shift method and antisolvent techniques in order to increase its dissolution rate. Various stabilizers were used, namely, Tween 20 and 80, HPMC, PVP K44, PVA, PEG 400, NaCMC, and $\beta$-cyclodextrin. The prepared nanosuspensions were characterized by Fourier transform infrared spectroscopy (FTIR) and scanning electron microscope (SEM) and evaluated for their particle size, particle size distribution, and in vitro dissolution rate. In general, it was found that the antisolvent method for the preparation of ET nanosuspensions reduced the drug particle size to a higher extent compared to the $\mathrm{pH}$ shift method. The dissolution rate of ET in distilled water was markedly enhanced in the nanosized system, as more than $65 \%$ of drug dissolved in $10 \mathrm{~min}$ from all the nanosuspension formulations except F5 (stabilized with PVP K44) and F8 (stabilized with Tween 20), as compared to less than $20 \%$ of crude drug. Nanoparticles prepared by antisolvent method using Tween 80 as a stabilizer were selected for further in vivo study. The in vivo test demonstrated that nanoparticles of ET were well absorbed with a percentage drug absorption value 2.7 times more than that of micrometric size of crude ET.
\end{abstract}

\section{Introduction}

Formulation of a poor water soluble drug has always been a challenging problem to pharmaceutical industry $[1,2]$. There are many conventional methods that have been reported for improving solubility of poorly soluble drugs. Among these methods are micronization [3], solubilization using cosolvents [4], solid dispersions [5], and precipitation [6]. The limitation of this precipitation technique is that the drug needs to be soluble in at least one solvent and this solvent needs to be miscible with antisolvent. Moreover, precipitation technique is not applicable to drugs, which are simultaneously poorly soluble in aqueous and nonaqueous media $[7,8]$.

Nanosuspensions are colloidal dispersions and biphasic system consisting of drug particles dispersed in an aqueous medium in which the diameter of the suspended particles is less than $1 \mu \mathrm{m}$ in size $[9,10]$. Nanosuspension can be produced by an appropriate size-reduction method and stabilized by a suitable stabilizer $[8,11,12]$.

According to Noyes-Whitney and Ostwald-Freundlich principles, the particle size in the nanometer range can lead to increased dissolution velocity and saturation solubility for a nanosuspension, which is usually accompanied by an increase in bioavailability $[8,13,14]$.

Nanosuspensions can be prepared by two methods, namely, "bottom up technology" and "top down technology" [15]. Bottom up technology is a method to form nanoparticles like precipitation, microemulsion, and melt emulsification methods. Top down technology involves the disintegration of larger particles into nanoparticles, examples of which are high-pressure homogenization and milling methods [16-18].

In bottom up technology, the nanoprecipitation method presents numerous advantages, as being a straightforward technique and being rapid and easy to perform. The drug is dissolved in a solvent, which is then added to nonsolvent 
that causes precipitation of the fine drug particles [19]. Nanosuspension of Danazol, Naproxen, and zaltoprofen has been prepared by precipitation technique to improve their dissolution rate and oral bioavailability $[1,20,21]$. The solubility and dissolution rates as well as the antioxidant of curcumin nanoparticles prepared by antisolvent method were significantly higher than those of the original curcumin [22]. Nitrendipine, furosemide, and acyclovir nanosuspensions to enhance the drug dissolution rate and oral bioavailability were prepared by the precipitation-ultrasonication method [23-25]. Controlled nanoprecipitation by $\mathrm{pH}$-shift method has been also reported $[8,26]$.

Etodolac (ET) is a nonsteroidal anti-inflammatory (NSAI) drug prescribed for the treatment of acute pain, osteoarthritis, and rheumatoid arthritis at an oral dose of $200 \mathrm{mg}$ twice daily [27-30]. Recent studies have proved that ET has antitumor effect on different human cancer cells $[31,32]$. ET is one of the selective COX-2 inhibitors; it possesses 10 -fold COX-2 selectivity over COX-1 [32, 33]. COX-2 inhibitors are responsible for the production of prostaglandins which is involved in cytoprotection of gastric mucosa and regulation of the renal blood flow. Thus, ET safely treats inflammatory disorders without causing gastric irritation, ulceration, or bleeding [33, 34].

ET is administered as a racemate. Both enantiomers are stable and there is no evidence of $R$ - to $S$-conversion in vivo [35]. Similar to other NSAIDs, the drug is highly plasma protein bound ( $>99 \%$ bound, primarily to albumin) and undergoes virtually complete biotransformation to oxidized metabolites and acyl-glucuronides. ET is well absorbed, with maximal plasma concentrations attained within 1 to 2 hours in healthy volunteers. The area under the plasma concentration-time curve of racemic ET increases linearly with doses used clinically. The elimination half-life of ET is between 6 and 8 hours in plasma and is similar for both enantiomers.

Depending on the biopharmaceutical classification system [36], dissolution rate is the rate-controlling step in the absorption process for drugs possessing high membrane permeability but low aqueous solubility (class II drugs). ET is practically water insoluble $(75 \mu \mathrm{g} / \mathrm{mL})$ and is belonging to class II drugs [37]. Thus, its oral bioavailability is expected to be limited by its dissolution rate, which might be increased using nanosuspension technology.

In the present study, nanosuspensions of ET were prepared by two different methods, namely, pH-shift method and antisolvent method. Different stabilizers were utilized for their steric stabilization character such as polyvinylpyrrolidone (PVP), hydroxypropyl methylcellulose (HPMC), polyvinyl alcohol (PVA), beta-cyclodextrin ( $\beta$-cyclodextrin), sodium carboxymethyl cellulose ( $\mathrm{Na} \mathrm{CMC}$ ), polyoxyethylene 20 sorbitan monolaurate (Tween 20 ), polyoxyethylene 80 sorbitan monolaurate (Tween 80 ), and polyethylene glycol 400 (PEG-400). Furthermore, the nanosuspension that showed promising results concerning the particle size and dissolution rate were subjected to in vivo pharmacokinetic study in rats.

\section{Experimental}

2.1. Experimental Materials. ET of $99.2 \%$ purity, (AMSAS.P.A, Italy), was kindly supplied by (Alamryia Pharmaceuticals, Alexandria, Egypt), hydroxypropyl methylcellulose (HPMC) (viscosity $6 \mathrm{cps}$ ) by (Colorcon, U.K.), sodium carboxymethyl cellulose (Na CMC) by (Elnasr Pharmaceutical Chemicals Co., Egypt), polyvinyl alcohol (PVA) by (Alfa Aesar, Germany), $\beta$-cyclodextrin by (Fluka, Germany), polyvinylpyrrolidone (PVP) by (Win Lab., UK), Tween 20 and Tween 80 by (Sigma, Germany), and polyethylene glycol 400 (PEG 400) by (European Co. For Pharmaceutical Industries, Egypt). All other reagents are of analytical grade.

\subsection{Methods}

2.2.1. Preliminary Studies. Preliminary studies were carried out to determine the most promising drug to stabilizer ratio (in terms of weight) that produces nanosuspensions with least aggregation. Different ratios of drug to stabilizer (9:1 to $1: 1)$ were examined.

\subsubsection{Preparation of ET Nanosuspensions by $p H$ Shift Method.} Calculated amount of the drug was weighed and dissolved in an appropriate volume of $0.1 \mathrm{~N} \mathrm{NaOH}(22.5 \mathrm{mg} / \mathrm{mL})$ containing the calculated amount of the stabilizer used. The drug to polymer ratio $(3: 1)$ was kept constant in all formulations. The solution's $\mathrm{pH}$ was reduced from 12 to 5.5 within $5 \mathrm{~min}$ by the addition of $0.1 \mathrm{~N} \mathrm{HCl}$ [38]. By reducing the $\mathrm{pH}$ of the solution, nanosuspensions were formed spontaneously due to low solubility of ET $(\mathrm{pKa}=4.5)$ at low $\mathrm{pH}$. The same procedure was carried out to prepare drug nanosuspension without stabilizer for comparison and denoted as F2. Crude drug was denoted by F1. Eight stabilized formulations were prepared by $\mathrm{pH}$ shift method and denoted as F3: F10 (Table 1).

\subsubsection{Preparation of ET Nanosuspensions by Antisolvent} Method. Calculated amount of the drug was weighed and dissolved in $5 \mathrm{~mL}$ ethyl alcohol. The organic solution of the drug was added at once to $10 \mathrm{~mL}$ of water containing the calculated amount of water soluble stabilizer under stirring at $1200 \mathrm{rpm}$ using magnetic stirrer at room temperature. The drug to polymer ratio $(3: 1)$ was kept constant for all formulations. By adding the organic solution of the drug to water, nanosuspensions were formed spontaneously due to the poor solubility of the drug in water. The same procedure was carried out to prepare drug nanosuspension without stabilizer for particle size comparison study and denoted as F2*. Eight stabilized formulations were prepared by antisolvent method and denoted as F11 : F18 (Table 2).

2.2.4. Drying of the Prepared Nanosuspensions. The prepared nanosuspensions were cooled rapidly to $-70^{\circ} \mathrm{C}$ and stored overnight. Freeze-drying of the samples was performed with a Telstar freeze-dryer (Terrassa, Spain) at a temperature of $-50^{\circ} \mathrm{C}$ with a pressure below $1 \mathrm{mbar}$; then the vials were removed after $48 \mathrm{hr}$ of drying. The samples were stored in well-closed vials at $-20^{\circ} \mathrm{C}$ for further investigations $[15,22]$. 
TABLE 1: Drug content, percentage yield, particle Size (P.S.), and size distribution of nanosuspensions prepared by pH shift method.

\begin{tabular}{|c|c|c|c|c|c|}
\hline Formula code & Stabilizer & $\begin{array}{l}\text { Drug content (\%) } \\
\text { pH shift method }\end{array}$ & $\begin{array}{c}\text { \% Yield } \\
\text { pH shift method }\end{array}$ & P.S. in nm & $\mathrm{PdI}^{*}$ \\
\hline F1 & Crude drug & - & - & $3676 \pm 42.6$ & 0.52 \\
\hline $\mathrm{F} 2$ & Nanosuspension without stabilizer & 100 & 99.46 & $7129 \pm 63.2$ & 1.00 \\
\hline F3 & $\mathrm{Na} \mathrm{CMC}$ & 98.7 & 92.02 & $827 \pm 5.4$ & 0.49 \\
\hline $\mathrm{F} 4$ & HPMC & 89.5 & 90.91 & $1025 \pm 11.14$ & 0.56 \\
\hline F5 & PVP K44 & 85.3 & 93.75 & $1702 \pm 97.2$ & 0.72 \\
\hline F6 & PVA & 90.0 & 96.77 & $2000 \pm 50.7$ & 0.85 \\
\hline F7 & PEG 400 & 92.6 & 95.54 & $790 \pm 3.3$ & 0.51 \\
\hline F8 & Tween 20 & 95.7 & 90.91 & $618 \pm 4.5$ & 0.49 \\
\hline F9 & Tween 80 & 92.5 & 90.36 & $393 \pm 2.05$ & 0.34 \\
\hline F10 & $\beta$-cyclodextrin & 98.2 & 96.77 & $866 \pm 5.9$ & 0.53 \\
\hline
\end{tabular}

PdI $^{*}$ : polydispersibility index.

TABLE 2: Drug content, percentage yield, particle Size (P.S.), and size distribution of nanosuspensions prepared by antisolvent method.

\begin{tabular}{|c|c|c|c|c|c|}
\hline Formula code & Stabilizer & $\begin{array}{l}\text { Drug content (\%) } \\
\text { antisolvent method }\end{array}$ & $\begin{array}{c}\text { \% Yield } \\
\text { antisolvent method }\end{array}$ & P.S. in nm & $\mathrm{PdI}^{*}$ \\
\hline F1 & Crude drug & - & - & $3676 \pm 42.6$ & 0.52 \\
\hline $\mathrm{F} 2$ & Nanosuspension without stabilizer & 100 & 99.74 & $4234 \pm 67.4$ & 1.00 \\
\hline F11 & $\mathrm{Na} \mathrm{CMC}$ & 92 & 85.77 & $464 \pm 7.6$ & 0.83 \\
\hline F12 & HPMC & 96.8 & 98.33 & $603 \pm 5.9$ & 0.78 \\
\hline $\mathrm{F} 13$ & PVP K45 & 80.0 & 87.92 & $290 \pm 6.8$ & 0.85 \\
\hline F14 & PVA & 85.5 & 91.93 & $468 \pm 9.1$ & 0.85 \\
\hline F15 & PEG 400 & 90.0 & 92.86 & $474 \pm 6.2$ & 0.78 \\
\hline F16 & Tween 20 & 98.0 & 93.09 & $119 \pm 4.6$ & 0.79 \\
\hline F17 & Tween 80 & 95.0 & 92.80 & $160 \pm 3.9$ & 0.54 \\
\hline F18 & $\beta$-cyclodextrin & 95.0 & 93.61 & $517 \pm 7.4$ & 0.78 \\
\hline
\end{tabular}

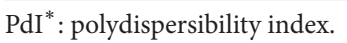

2.2.5. Fourier Transform Infrared Spectroscopy (FTIR). FTIR spectra were performed using Perkin-Elmer FTIR series (model-1615) spectrophotometer to find out any possible drug-stabilizer interactions. Samples of ET and of each stabilizer used (PVP, HPMC, PVA, $\beta$-Cyclodextrin, Na CMC, Tween 20, Tween 80, and PEG-400) as well as samples of the prepared nanosuspensions were grounded separately and mixed thoroughly with potassium bromide. The ratio of sample and $\mathrm{KBr}$ was kept for all the preparations. The potassium bromide discs were prepared by compressing the powders at a pressure of 5 tones for $5 \mathrm{~min}$ in a hydraulic press. Scans were obtained from 4000 to $500 \mathrm{~cm}^{-1}$.

2.2.6. Scanning Electron Microscopy (SEM). Morphologies of raw ET and nanosized ET were examined using a scanning electron microscope (JEOL JSM-7001F, Japan) operated at an accelerating voltage of $1 \mathrm{KV}$ and a secondary detector. Freshly prepared ET nanosuspensions and dispersion of raw ET were deposited on a glass slide following evaporation of solvent.

2.2.7. Particle Size Determination of Nanosuspensions. Particle size and size distribution of the prepared nanosuspensions were measured using a dynamic light scattering particle size analyzer, model Zetasizer Nano-ZS; model number ZEN 3500, Malvern (UK). Raw data were collected over 5 minutes at $25^{\circ} \mathrm{C}$ and at an angle of 90 degrees and further processed using the ZPW388 software program. Nanosuspensions were diluted 50 -fold in deionized water before measurement.

2.2.8. Determination of Drug Content in Lyophilized Samples. ET content in lyophilized nanosuspensions' samples was analyzed by dissolving $10 \mathrm{mg}$ of each sample separately in $5 \mathrm{~mL}$ ethanol. The samples were vortexed for $1 \mathrm{~min}$ at room temperature and filtered $(0.45 \mu \mathrm{m}$, Millipore) and one $\mathrm{mL}$ was suitably diluted with water. The amount of drug was determined spectrophotometrically (Perkin Elmer, Japan) at $279 \mathrm{~nm}$ against water as blank.

2.2.9. Physical Appearance. To evaluate the physical appearance, nanosuspension samples were observed for agglomeration and color change for two weeks at room temperature after preparation.

2.2.10. In Vitro Release Study. Lyophilized samples equivalent to $50 \mathrm{mg}$ of ET were used to study the drug release rate 
using USP dissolution apparatus II (Erweka, Germany). Lyophilized powder was dispersed in $500 \mathrm{~mL}$ of either water or phosphate buffer $\mathrm{pH} 6.8$ at $37 \pm 0.5^{\circ} \mathrm{C}$ to maintain sink conditions. Stirring rate was kept constant at $100 \mathrm{rpm}$. At predetermined time intervals, $5 \mathrm{~mL}$ aliquots were withdrawn and filtered $(0.45 \mu \mathrm{m}$, Millipore $)$ and the amount of dissolved ET was determined spectrophotometrically at $279 \mathrm{~nm}$. Dissolution rate of an amount of $50 \mathrm{mg}$ of crude drug in $500 \mathrm{~mL}$ of either water or phosphate buffer 6.8 was determined and used as reference for comparison. Each dissolution experiment was performed in triplicates and the average values and standard deviations were calculated. Calculation of dissolution efficiency (DE) has been applied for the evaluation of pharmaceutical equivalence between formulations. Dissolution efficiency (DE) was calculated from the area under the dissolution curve at time $t$ (measured using the trapezoidal rule) and expressed as percentage of the area of the rectangle described by $100 \%$ dissolution in the same time,

$$
\% \text { Dissolution efficiency }(\mathrm{DE})=\left[\frac{\int_{t}^{y} d t}{y(100) t}\right] \times 100 \text {. }
$$

\subsection{In Vivo Absorption Study}

2.3.1. Animals. Male Albino Wister rats were provided by the laboratory Animal Center, King Saud University, Riyadh, Saudi Arabia. The study was performed in accordance with the guidelines of the local institutional animal ethics committee.

2.3.2. Dosing and Samples Collection. Bioavailability of two preparations (F1 \& F17) was assessed in rats. Twelve rats, weighing $0.25-0.3 \mathrm{~kg}$, were divided into two groups randomly (six animals each) and given ET orally with gastric catheter at a single dose of $20 \mathrm{mg} / \mathrm{kg}$ after fast overnight but were allowed free access to water. Orbital sinus blood (approximately $2 \mathrm{~mL}$ ) was collected into tubes containing $2.6 \mathrm{mmol}$ sodium edetate before and at 15 and $30 \mathrm{~min}$ and 1, 3, 5, 12, 24, and $48 \mathrm{hr}$ after drug administration. Plasma was separated by centrifugation for $15 \mathrm{~min}$ at $10000 \mathrm{rpm}$ at $4^{\circ} \mathrm{C}$ using $3-30 \mathrm{k}$ centrifuge (Sigma, Germany). The specimens were stored at $-70^{\circ} \mathrm{C}$ until analysis [35]. The concentration of ET in rat plasma was determined by HPLC.

2.3.3. HPLC Assay Method. Plasma samples were analyzed for ET using reported HPLC method with some modifications [39], where hydrochlorothiazide was used as internal standard (IS). Calibration curves of ET were prepared with volumes of $300 \mu \mathrm{L}$ of rat plasma spiked with ET and the IS to yield final ET concentrations of $0.1,0.5,1.0,5.0,10$, $25,50,75$, and $100 \mu \mathrm{g} \mathrm{mL}^{-1}$ of ET and IS $\left(10 \mu \mathrm{g} \mathrm{mL}^{-1}\right)$ in disposable Falcon $15 \mathrm{~mL}$ conical centrifuge tubes (Thermo Fisher Scientific Inc., MA, USA). Each tube was vortexed for $1 \mathrm{~min}$ and was then mixed with $300 \mu \mathrm{L}$ of $5 \%$ formic acid for deproteinization. Analytes were extracted with $5 \mathrm{~mL}$ ethyl acetate and centrifuged at 5,000 rpm for $10 \mathrm{~min}$. The supernatant layer was separated and evaporated to dryness under a gentle steam of nitrogen using the 27 port Reacti-Vap evaporator (Thermo Fisher Scientific Inc., MA, USA). The residue was reconstituted in $1 \mathrm{~mL}$ mobile phase and filtered through a $0.45 \mu \mathrm{m}$ Millex filter (Millipore, Bedford, MA, USA). Blank rat plasma samples were processed in the same manner using water rather than ET and IS. The accuracy and precision of the method was calculated. The peak area ratios of ET and IS and their concentrations were used as the basis for the quantification.

Reagents and Solvents. HPLC water was purified using cartridge system (Milford, Bedford, USA). Ultrapure water with resistivity of $18.2 \mu \Omega-\mathrm{cm}$ was obtained from Milli-Q plus purification system (Millipore, Bedford, MA, USA). Acetonitrile and ethyl acetate HPLC grade was purchased from $\mathrm{BDH}$ laboratory supplies (Poole-UK). All other reagents used were of analytical grade and purchased from Sigma (St. Louis, MO, USA).

2.3.4. HPLC System and Chromatographic Conditions. HPLC analysis was carried out on a Waters Breeze system (Waters Corporation, Milford, MA, USA) equipped with 1525 binary pump with on-line degasser, $717+$ autosampler, $5 \mathrm{CH}$ thermostated column compartment, and 2487 UV dual wavelength absorbance detector. The chromatographic separations were performed on Symmetry C18 column $(3.5 \mu \mathrm{m}$, $75 \mathrm{~mm} \times 4.6 \mathrm{~mm}$ i.d) manufactured by (Waters Corporation, Milford, MA, USA). The column temperature was kept constant at $25 \pm 2^{\circ} \mathrm{C}$. Separations were performed in isocratic mode, with a mobile phase consisted of $0.01 \mathrm{M}$ potassium dihydrogen phosphate ( $\mathrm{pH} 7.4$ adjusted with $0.1 \mathrm{M} \mathrm{KOH}$ ) and acetonitrile $(70: 30, \mathrm{v} / \mathrm{v})$ pumped at flow rate of $1.0 \mathrm{~mL} / \mathrm{min}$. The mobile phase was filtered by a Millipore vacuum filtration system equipped with a $0.45 \mu \mathrm{m}$ pore size filter. The samples (10 $\mu \mathrm{L}$ each) were injected into the HPLC system. The highest sensitivity for both ET and IS was achieved when the detector was set at $235 \mathrm{~nm}$. Under these optimum chromatographic conditions, ET and IS were detected at retention times of 2.66 and $1.47 \mathrm{~min}$, respectively; the total chromatographic run time was $4 \mathrm{~min}$.

2.3.5. Pharmacokinetics and Statistical Analysis. The mean best-fit linear regression equation was used to estimate the concentrations of ET at different time. All pharmacokinetic parameters were calculated using the pharmacokinetic software WinNonlin (version 5.2, Pharsight Corp., Mountain View, CA, USA) using noncompartmental method. The maximum plasma concentration $\left(C_{\max }\right)$ and the time to reach $C_{\max }\left(T_{\max }\right)$ were directly obtained from the plasma concentration-time curves. For each formulation, mean percentage absorbed-time plots were obtained by deconvolution of the corresponding mean concentration versus time plots profiles using Wagner-Nelson method [40]. These plots allowed the construction of percentage unabsorbed time plots, which were used for the calculation of the absorption rate constants [41]. Consider

$$
A_{t}=\frac{\left(C_{t}+K_{\mathrm{el}} \times \mathrm{AUC}_{0-t}\right)}{\left(K_{\mathrm{el}} \times \mathrm{AUC}_{0-\infty}\right)} .
$$


$A_{t}$ is the fraction of drug absorbed at time $t, C_{t}$ is the concentration of drug in the plasma at time $t, K_{\mathrm{el}}$ is the elimination rate constant, $\mathrm{AUC}_{0-t}$ is the area under the curve from time zero to time $t$, and $\mathrm{AUC}_{0-\infty}$ is the area under the plasma curve from time zero to infinity.

The elimination rate constant $\left(K_{\mathrm{el}}\right)$ was determined by simple linear regression based on the terminal log-linear part of the plasma concentration-versus-time profile. The apparent elimination half-life $\left(t_{1 / 2}\right)$ was calculated as $0.693 / K_{\mathrm{el}}$.

2.4. Statistical Analysis. All the results were expressed as mean values \pm standard deviation $(\mathrm{SD})$. One-way analysis of variance (ANOVA) with Turkey's multiple comparisons post hoc was used to test for significance, at a $5 \%$ significance level. Statistical difference dealing $(P<0.05)$ were considered significant [42].

\section{Results and Discussion}

3.1. Considerations for Selection of Stabilizers. Manufacturing of nanosuspensions involves the generation of a large number of small particles with enormous surface area. This significantly increases the Gibb's free energy of the system and, due to the high interfacial tension, these systems are thermodynamically unstable. Accordingly, nanoparticles will tend to minimize their total energy by undergoing agglomeration [43].

The process of agglomeration depends on the activation energy, which is influenced by the addition of stabilizers to the system (such as, surfactants and polymers). These stabilizers reduce the interfacial tension between the particles and the dispersion medium and act as wetting agents. The second requirement is to provide a barrier between the drug particles to prevent agglomeration by electrostatic attraction (ionic surfactants) or steric stabilization (nonionic surfactants and polymers) [43].

It is well known that an appropriate stabilizer is very important to control particle growth during the production of uniform nanoparticles. Many reports have shown that if preliminary particles can be arrested efficiently by appropriate stabilizers, the nanosuspensions system can be maintained for a longer time [44].

The adsorption properties of stabilizers can be affected by the nature of stabilizer and drug surface, for example, molecular weight is an important factor for polymeric stabilizers. The chain length should be high enough, so that polymers chains have an optimum length to overcome the Van der Waals forces of attraction. Furthermore, another important factor is the size of the polymer [45].

Electrolytes are present in the gastrointestinal tract and the contact of the nanocrystals with these electrolytes cannot be avoided. Electrostatic stabilization is reduced in its efficiency in an electrolyte containing environment. To compensate for this it is ideal to use steric stabilizers, which are less impaired in their effect by electrolytes [43].

The adsorption layer of the stabilizer shifts the plain of shear, at which the zeta potential is measured, to a larger distance from the particle surface. Consequently the measured zeta potential is lower. In such cases zeta potentials of about $20 \mathrm{mV}$ are still sufficient to fully stabilize the system [46]. Generally, absolute zeta potential value higher than $60 \mathrm{mV}$ is considered of extreme stability, $30 \mathrm{mV}$ means good stability, $20 \mathrm{mV}$ shows acceptable short term stability and less than $5 \mathrm{mV}$ will induce fast particle aggregation [43]. However, this rough guideline is only viable to pure electrostatic stabilization or in combination with low-molecular weight surfactants [43]. In this study Zeta potential was not determined since all stabilizer used act by steric stabilization.

Based on the above consideration various surfactants and polymers which exhibit steric stabilization characteristics were preferably used in this study such as Tween 20, Tween 80, HPMC, PVP K30, PVA, PEG 400, Na CMC, and $\beta$ cyclodextrin. The selected stabilizers were screened by trials in terms of their performance and particle sizes. It was found that nanosuspension prepared using drug to stabilizer ratio of 9:1 produced large particle size and rapid precipitation was noticed. Increasing the ratio of stabilizer in the nanosuspension to $9: 3(3: 1)$ resulted in a dramatic reduction in particle size without precipitation of the formed nanosuspension. Following a further increase in stabilizer concentration, the particle size was not markedly reduced, which indicated that the drug particle surface was already sufficiently enveloped by the stabilizer molecules.

In conclusion, a preliminary study revealed that drug to stabilizer ratio of $3: 1$ was of choice in terms of performance and particle size.

3.2. Fourier Transform Infrared Spectroscopy (FTIR). FTIR spectroscopy Figures 1(a)-1(h) revealed that there is neither appearance of new peaks nor disappearance of existing peaks, which indicated that there is no interaction between the drug and each of the stabilizers used. The characteristic ketone $(\mathrm{C}=\mathrm{O})$ stretching vibration at $1747.22 \mathrm{~cm}^{-1}, \mathrm{C}-\mathrm{H}$ bending at $1412.57 \mathrm{~cm}^{-1}, \mathrm{C}-\mathrm{O}$ stretching at $1261.59 \mathrm{~cm}^{-1}, \mathrm{C}-\mathrm{N}$ vibration at $1313.29 \mathrm{~cm}^{-1}$, aromatic $\mathrm{C}-\mathrm{H}$ stretching at $747.69 \mathrm{~cm}^{-1}$ and the stretching band in the region of $3500-3200 \mathrm{~cm}^{-1}$ assigned to the nonbonded aromatic amino group were identified in all the spectrums of the lyophilized nanosuspensions.

3.3. Scanning Electron Microscopy (SEM). Pure drug and nanoparticles surface morphology and shape were analyzed by SEM, representative examples are shown in Figure 2. It can be seen that the raw drug particles existed as irregular tabular and prismatic crystals with smooth surface Figure 2(a). Micronized ET powder showed irregular shapes with particle size generally larger than the ET prepared nanoparticles and with a broad particle size distribution. The ET prepared nanoparticles were more uniform in shape as compared to pure drug but with more or less rough surfaces (Figures 2(b), 2(c) and 2(d)). The nanocrystals were found to be slightly cubical in shape with a narrow particle size distribution. This might be ascribed to the adsorption of stabilizer on the surface of the drug particle. The morphologies of ET nanoparticles obtained by $\mathrm{pH}$ shift method or antisolvent method using PVP as a stabilizer are shown in Figures 2(b) and 2(c), 

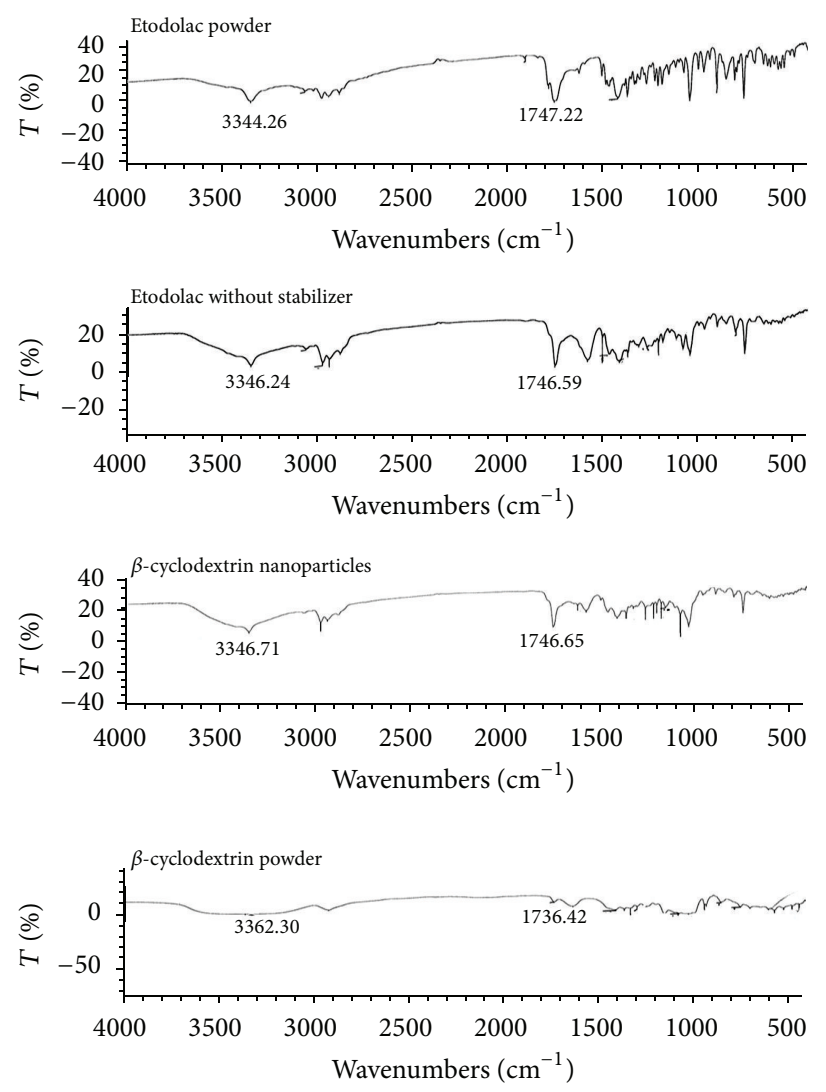

(a) IR spectra of ET nanosuspension stabilized with $\beta$-cyclodextrin
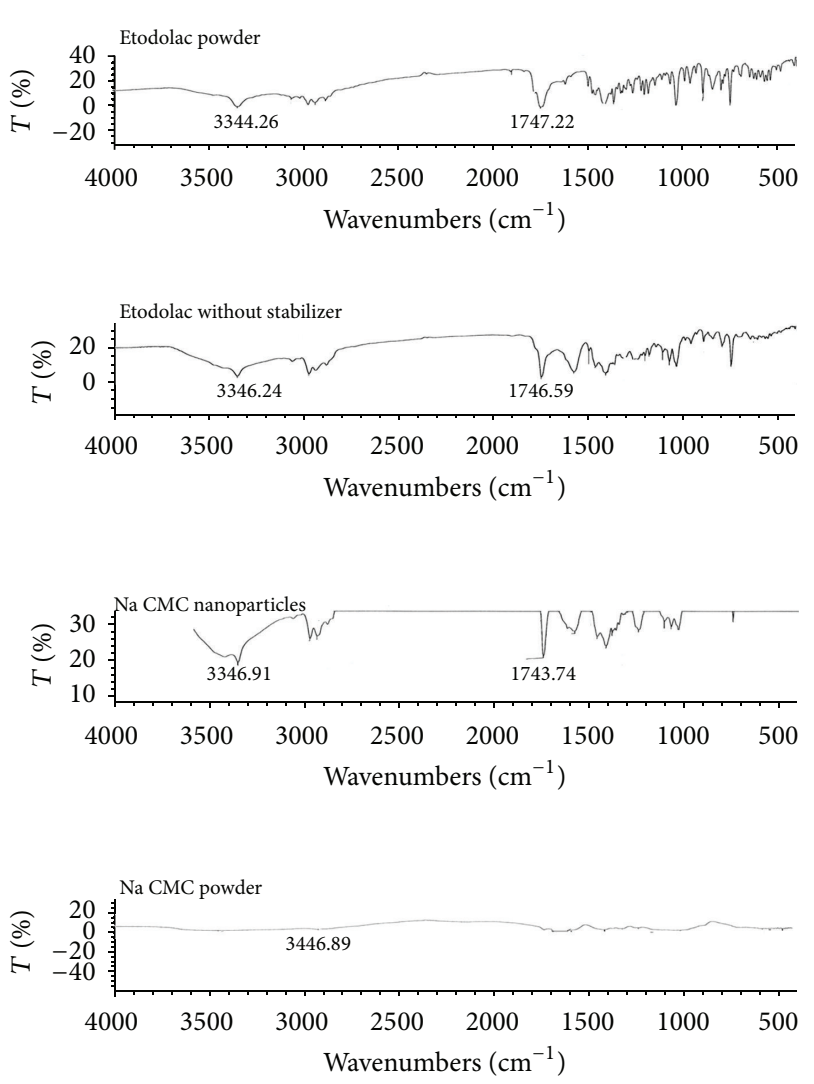

(c) IR spectra of ET nanosuspension stabilized with $\mathrm{Na}$ CMC
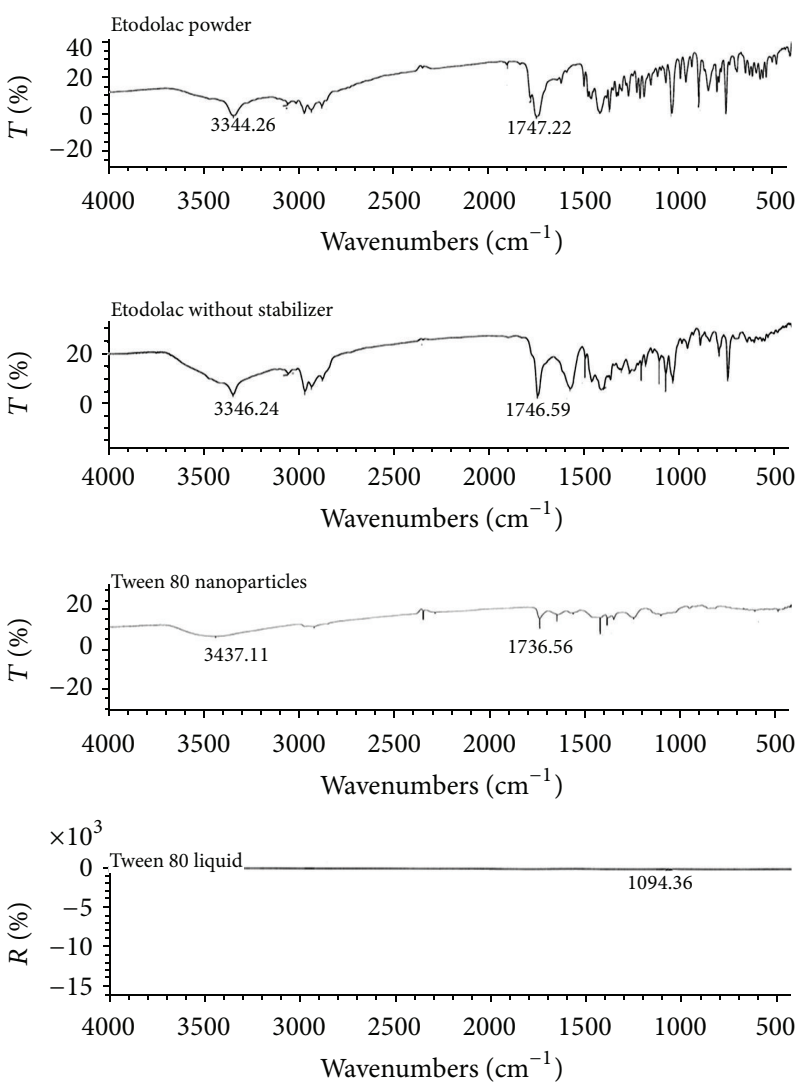

(b) IR spectra of ET nanosuspension stabilized with Tween 80
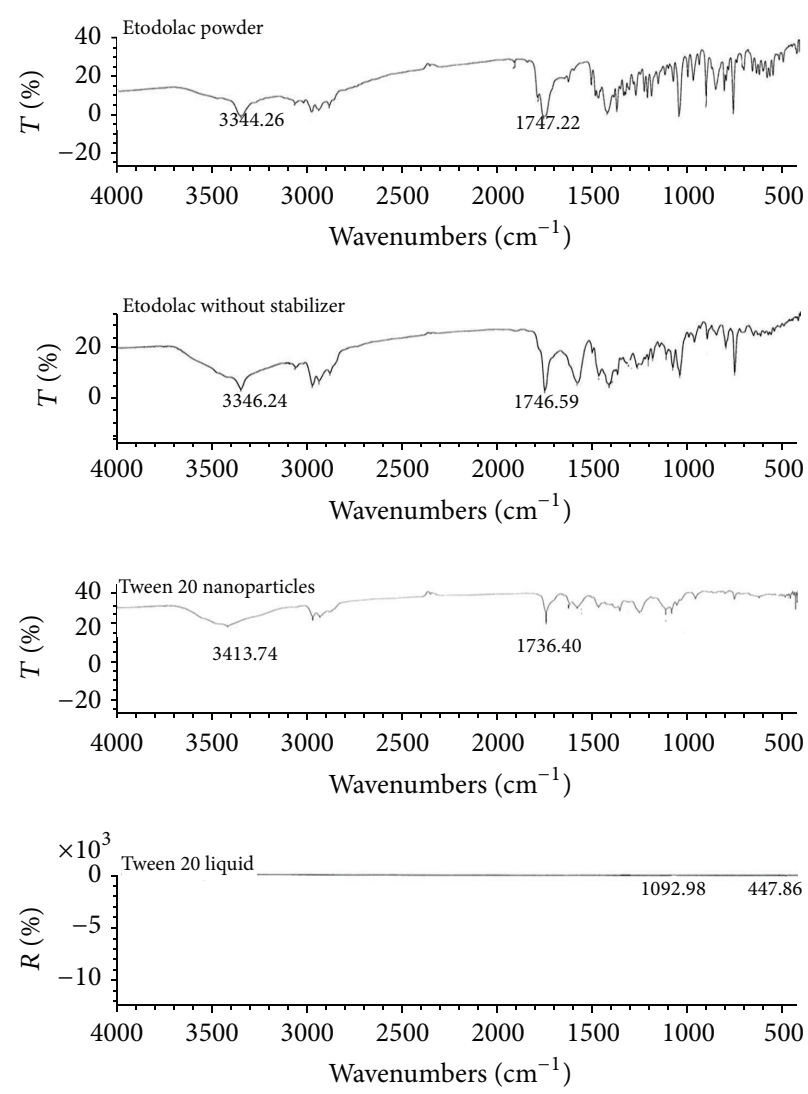

(d) IR spectra of ET nanosuspension stabilized with Tween 20

Figure 1: Continued. 

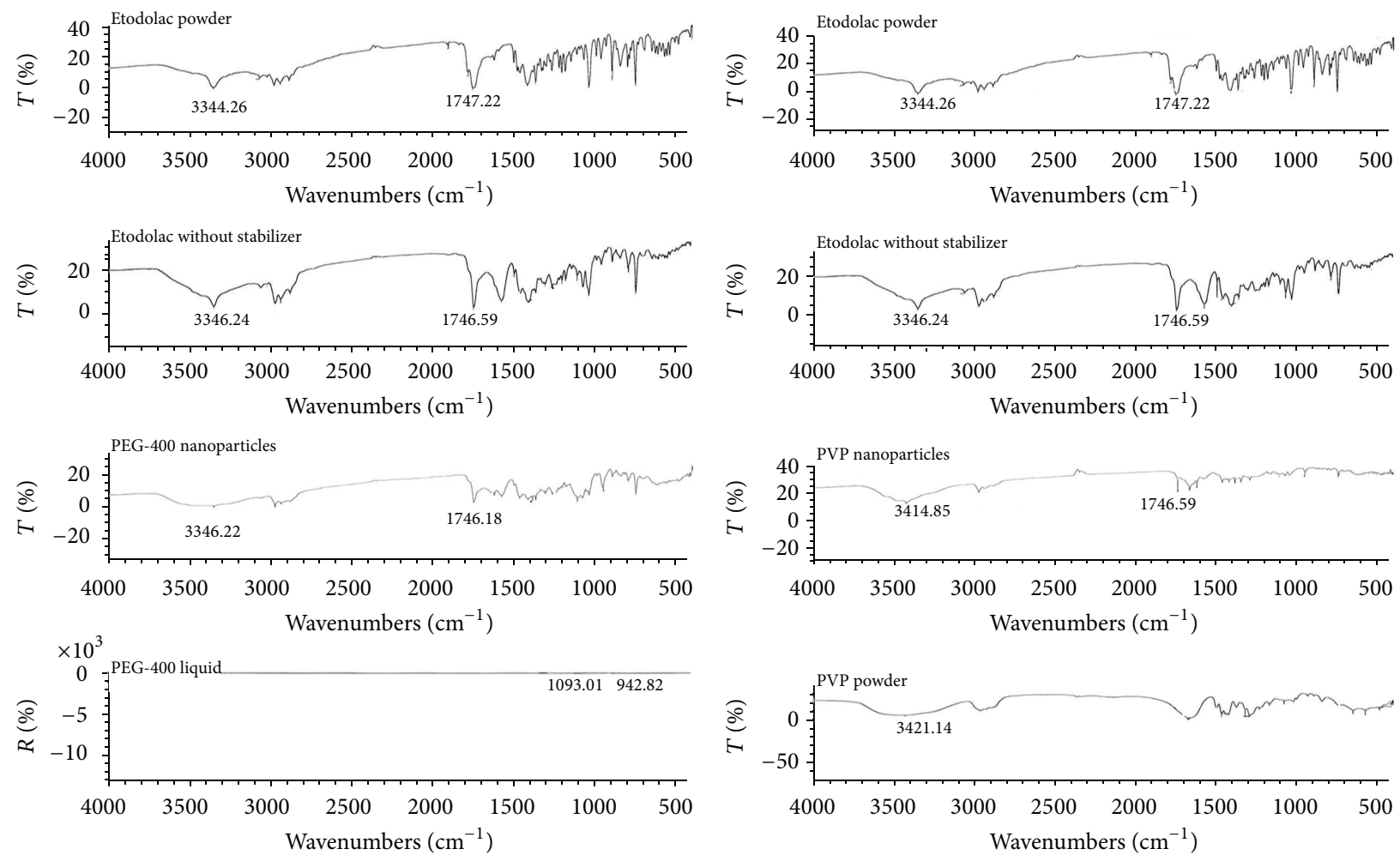

(e) IR spectra of ET nanosuspension stabilized with PEG 400

(f) IR spectra of ET nanosuspension stabilized with PVP K44
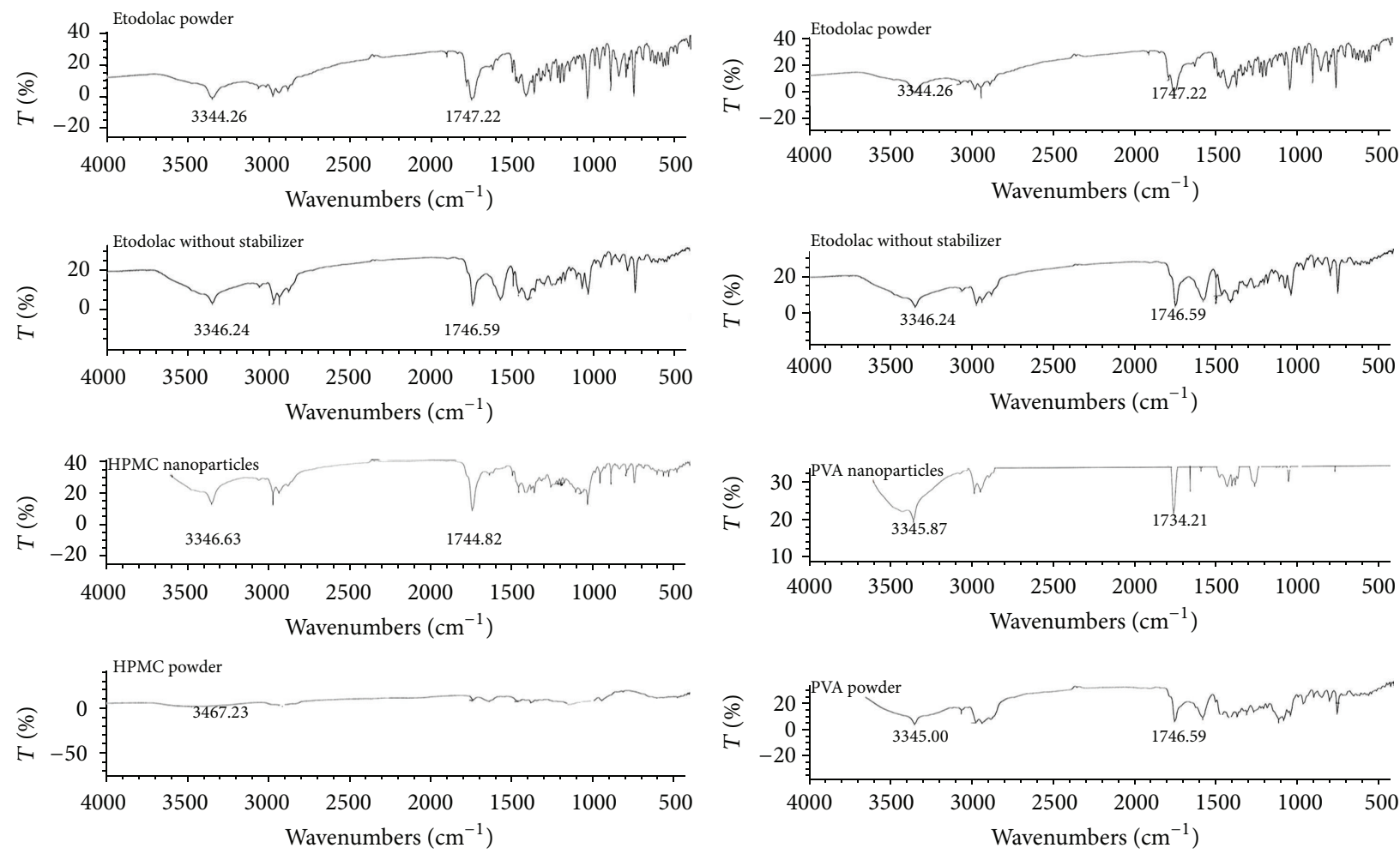

(g) IR spectra of ET nanosuspension stabilized with HPMC

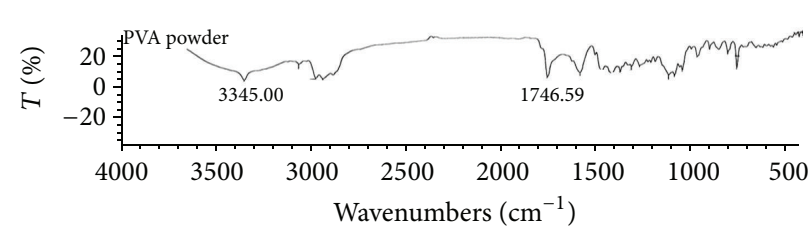

(h) IR spectra of ET nanosuspension stabilized with PVA

FIGURE 1: Fourier transformed infrared (FTIR) spectra of pure drug, drug nanosuspension, pure stabilizers, and all nanosuspensions formulations. 


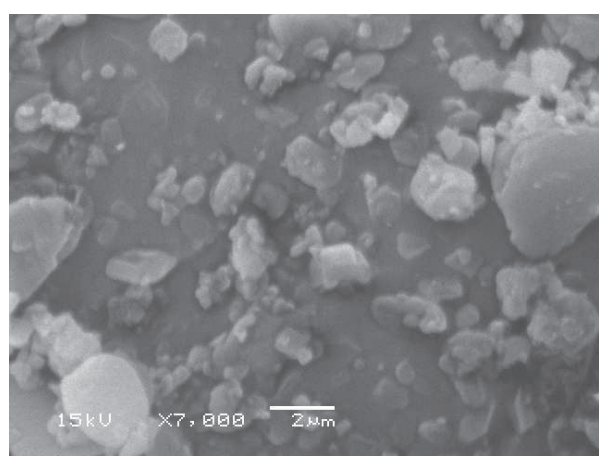

(a)

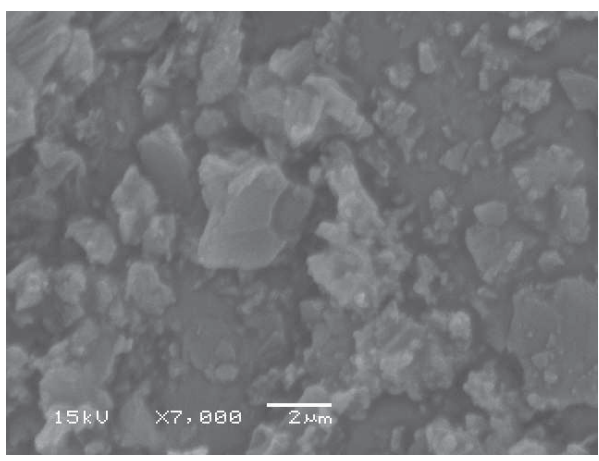

(c)

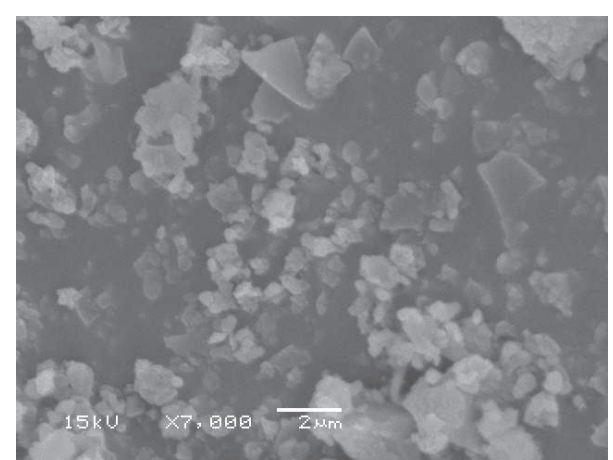

(b)

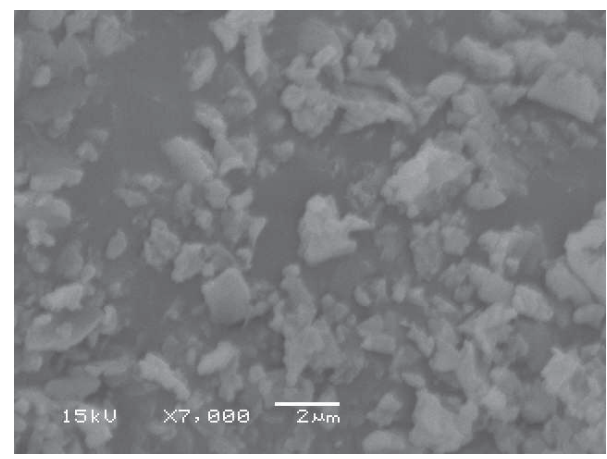

(d)

FIgURE 2: Photomicrographs of SEM of pure ET powder (a), pH shift method using PVP as a stabilizer (b), antisolvent method using PVP as a stabilizer (c), and $\mathrm{pH}$ shift method using Tween 80 as a stabilizer (d).

respectively. Morphology of nanoparticles prepared by $\mathrm{pH}-$ shiflt method using Tween 80 as a stabilizer is presented in Figure 2(d). The images revealed the presence of aggregates or particle assemblies which were composed of a large number of individual nanoparticles. The appearance of aggregates was ascribed to the dilution effect during redispersion process. The redispersion displaced part of adsorbed layer of the stabilizer from the nanocrystals surfaces forming a thinner layer. The weakened steric hindrance effects, derived from the thinner stabilizer layer, would probably result in partial aggregation among nanocrystals, which would increase the particle size distribution of nanocrystals.

3.4. Physical Appearance. Visual examination of all prepared nanosuspensions was done on a basis of color change and agglomeration. There was no color change after lyophilization of nanosuspensions. Sedimentation of PVP, HPMC and PVA contained nanosuspension prepared by $\mathrm{pH}$ shift method occurred within 1-3 h. Large particle sizes (agglomerates) were observed. When we evaluated feasibility of nanosuspension production, poor performance was seen when the stabilizer was HPMC, increasing drug-to-stabilizer ratio did not result in stabilization of nanosuspension. It was noticed that when drug-to-stabilizer ratio was shifted from $3: 1$ to 2:1 for HPMC, more viscosity was imparted to the system compared to other stabilizers. More viscosity of HPMC was inherent disadvantage as it would slow down the process for nanosuspension [45].
Other stabilizers showed good stabilization as the nanosuspension did not sediment during the time of visual observation. Nanosuspensions prepared by antisolvent method using the same stabilizers were stable at room temperature for at least 4 days. These results may be attributed to the particle size and molecular weight (MW) of the stabilizer itself, in addition to the method of preparation [47].

3.5. Drug Content. During the preparation process there was no any drug loss step involved, so theoretically the formulation was considered as being 100\% drug content. Table 1, demonstrates the drug content for each nanosuspension formulation prepared. Formulations containing PVP as a stabilizer showed the least drug content on using the two different methods of preparation. Formulations containing $\beta$-cyclodextrin as a stabilizer and prepared by $\mathrm{pH}$ shift method showed the highest drug content (98.2\%) whereas, formulation containing Tween 20 as a stabilizer and prepared by antisolvent method had the highest drug content (98\%). It is obvious that the drug content of nanosuspension can be considered as a function of both the nature of the stabilizer used as well as the method of preparation. According to the data of Table 1, it can be seen that all the drug content were within acceptable limit.

3.6. Particle Size and Size Distribution Measurement. The mean particle size and the span of particle size distribution (polydispersity index, PI) are two important characteristic 
parameters because they affect the saturation solubility, dissolution rate, physical stability, even bioavailability of nanosuspensions. Particle size has primary impact on saturation solubility and ripening [48]. PI value of $0.1-0.25$ indicates a fairly narrow distribution whereas a PI value greater than 0.5 indicates a very broad distribution [49].

Particle size measurements of pure ET and its lyophilized nanosuspensions are listed in Tables 1 and 2 . The average particle size of ET crystals was $3676 \mathrm{~nm}(3.676 \mu \mathrm{m})$. As it can be seen, the size reduction by the two methods of nanosization are significant compared with the untreated powder of ET (Tables 1 and 2).

It was found that in absence of stabilizer the drug particle size increased from $3.676 \mu \mathrm{m}$ to 7.129 and $4.234 \mu \mathrm{m}$ for nanosuspensions prepared by $\mathrm{pH}$ shift and antisolvent method, respectively. The increase in particle size in absence of stabilizer was more pronounced in nanosuspension prepared by $\mathrm{pH}$ shift method in which particle size increased by 2 folds. Decrease in particle size might be a factor of stabilizer type as well as the method of preparation of nanosuspensions. In general, it is obvious that the anti-solvent method for the preparation of ET nanosuspension reduced the drug particle size to high extent compared to the $\mathrm{pH}$ shift method (Tables 1 and 2).

In solvent change method, the drug is firstly dissolved in a solvent. Then this solution is mixed with a miscible antisolvent in presence of surfactants or polymers. Rapid addition of a drug solution to the antisolvent (usually water) leads to sudden supersaturation of drug in the mixed solution, and generation of ultrafine crystalline or amorphous drug solids. This process involves two phases; nuclei formation and crystal growth. When preparing a stable suspension with a minimum particle size, a high nucleation rate but low growth rate is necessary. The major challenge is to avoid crystal growth due to Ostwald ripening being caused by different saturation solubility's in the vicinity of the differently sized particles [50]. High-supersaturation conditions are needed for rapid nucleation by adding drug in minimum volume of a water-miscible organic solution into the water under rapid mixing at low temperature. This rapid dilution with water, results in high-supersaturation conditions, and causes spontaneous nucleation and a subsequent reduction of the supersaturation condition in the vicinity of the nucleating crystals and reduction of crystal growth rates [50].

Particle size ranged from $119 \mathrm{~nm}$ for Tween 20 to $603 \mathrm{~nm}$ for HPMC, which seemed to be affected by relative viscosity of the polymeric dispersion in the presence of stabilizers and followed the trend: Tween $20>$ Tween $80>\mathrm{PVP}>\mathrm{NaCMC}>$ PVA $>$ PEG $400>\beta$-cyclodextrin $>$ HPMC. Nanosuspension with Tween as stabilizers possessed the smallest particle size while that containing HPMC had the largest particle size (Table 2).

It has been reported that particle-size distribution of solid particles in nanosuspensions is usually less than one micron with an average particle size ranging between $200-600 \mathrm{~nm}$ [18]. In the present study, nanosuspensions prepared by the antisolvent method showed particle size-distribution ranged from $0.539 \mu \mathrm{m}$ with an average particle size of $119 \mathrm{~nm}$ in case of Tween 20 to $0.850 \mu \mathrm{m}$ in case of PVP or PVA with an average particle size of $290 \mathrm{~nm}$ and $468 \mathrm{~nm}$, respectively (Table 2).

Tween 20 or 80 as stabilizers were adsorbed on the surface of the hydrophobic drug ET, a mechanical barrier was formed against crystal growth and agglomeration. The stabilizer occupied the adsorption sites on the surface of freshly formed drug crystals during process of crystallization. As a result, it inhibits subsequent growth by inhibiting the incorporation of drug molecules from solution into crystal lattices, as already observed for other drugs such as Felodipine and Folic acid [51, 52]. Moreover, Tween 20 and 80 being nonionic surfactants with small particles and low MW characteristics resulted in the production of nanosized particles of 119 and $160 \mathrm{~nm}$, respectively (Table 2 ).

HPMC is a large molecule, nonionic stabilizer and provides stability via steric stabilization. HPMC is a polymer containing a number of methoxyl and hydroxypropyl groups, and the hydrophobic parts have good affinity for drug particles and can be adsorbed onto the drug particle surface providing an effective steric barrier against growth. In addition, hydrogen bonds can be formed between the drug molecule and HPMC [44]. Because of its solution viscosity, it is adsorbed by a thick wide layer around drug particles thus increasing the particle size compared to the other polymers. HPMC is a polymeric stabilizer and polymer adsorption is known to be irreversible when compared to the more dynamic adsorption of small molecule stabilizers [44]. PVA-based formulation gave smaller nanoparticles than preparations based on HPMC as stabilizer which were a bit bigger [18].

It can be seen from Table 2 that nanosuspension prepared without stabilizer produced an average particle size $4234 \mathrm{~nm}$ with particle size distribution of one micron. Based on the above discussion, a significant particle size reduction was obtained by using a stabilizer. Therefore, it can be concluded at this stage of the study that, antisolvent method using drug to stabilizer ratio of $3: 1$ gave promising nanosuspensions in terms of their average particle size and particle size distribution.

As mentioned before, nanosuspensions prepared by $\mathrm{pH}$ shift method showed larger particle size than those prepared by antisolvent method (Tables 1 and 2). This could be due to the technique of nucleation. By changing the $\mathrm{pH}$ from 12 to 5.5 within 5 minutes, this condition generated low supersaturation resulting in slow nucleation rate and produced a small number of nuclei, which increased the solute mass for subsequent growth. Large nanoparticles in micron were obtained whereas others in the submicron were produced provided that the growth of nucleating crystals was inhibited by the stabilizer (surfactant or polymer). Nanosuspension containing PVA as a stabilizer showed the largest particle size $(2 \mu \mathrm{m})$ whereas; Tween 80 containing nanosuspension demonstrated the least particle size $(393 \mathrm{~nm})$.

3.7. Dissolution Rate Studies. The dissolution profiles of the drug release from the different nanosuspension formulations were illustrated in Figures 3-6. The dissolution behavior of ET and its nanosuspensions was expressed as percent 
TABLE 3: Dissolution parameters of ET nanosuspensions prepared by $\mathrm{pH}$ shift or antisolvent method in both distilled water and phosphate buffer (PB) pH 6.8 .

\begin{tabular}{|c|c|c|c|c|c|c|c|c|c|}
\hline \multirow{3}{*}{ Formula code } & \multicolumn{4}{|c|}{$\mathrm{pH}$ shift method } & \multirow{3}{*}{ Formula code } & \multicolumn{4}{|c|}{ Antisolvent method } \\
\hline & \multicolumn{2}{|c|}{ Distilled water } & \multicolumn{2}{|c|}{ PB pH 6.8} & & \multicolumn{2}{|c|}{ Distilled water } & \multicolumn{2}{|c|}{ PB pH 6.8} \\
\hline & $\mathrm{DR}_{10 \text { min }}$ & $\mathrm{DE}_{10 \min }$ & $\mathrm{DR}_{10 \text { min }}$ & $\mathrm{DE}_{10 \min }$ & & $\mathrm{DR}_{10 \text { min }}$ & $\mathrm{DE}_{10 \min }$ & $\mathrm{DR}_{10 \text { min }}$ & $\mathrm{DE}_{10 \min }$ \\
\hline F1 & 17.80 & 9.27 & 82.40 & 13.73 & $\mathrm{~F} 1$ & 17.8 & 9.27 & 82.40 & 13.73 \\
\hline F3 & 78.90 & 15.29 & 98.08 & 32.70 & F11 & 24.30 & 4.66 & 99.00 & 49.50 \\
\hline $\mathrm{F} 4$ & 79.90 & 26.63 & 77.65 & 17.26 & F12 & 84.50 & 15.09 & 99.00 & 49.50 \\
\hline F5 & 39.10 & 7.56 & 96.65 & 46.33 & F13 & 97.50 & 49.75 & 96.00 & 32.32 \\
\hline F6 & 75.23 & 12.71 & 83.70 & 15.67 & F14 & 57.60 & 9.80 & 93.60 & 20.80 \\
\hline F7 & 82.70 & 14.06 & 90.05 & 15.47 & F15 & 93.40 & 46.70 & 91.60 & 48.21 \\
\hline F8 & 52.00 & 9.24 & 67.90 & 11.85 & F16 & 95.20 & 47.60 & 91.20 & 31.93 \\
\hline F9 & 77.65 & 12.94 & 97.60 & 32.50 & F17 & 95.40 & 48.28 & 83.40 & 28.37 \\
\hline F10 & 96.00 & 48.00 & 98.08 & 31.80 & F18 & 107.7 & 4.39 & 90.50 & 31.16 \\
\hline
\end{tabular}

$\mathrm{F} 1=$ Crude drug.

$\mathrm{DR}_{10 \text { min }}$ : \% drug released after 10 min; DE: \% dissolution efficiency after $10 \mathrm{~min}$.

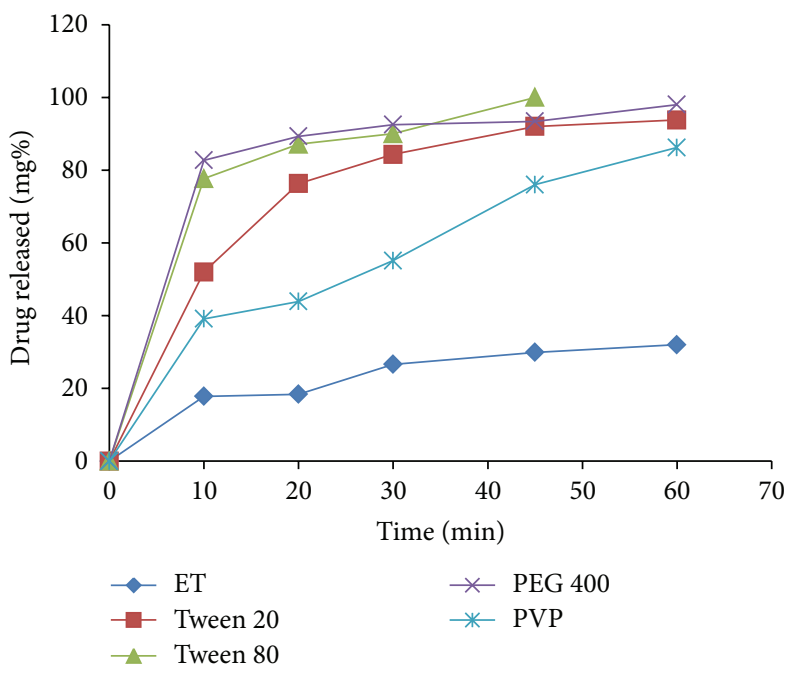

(a)

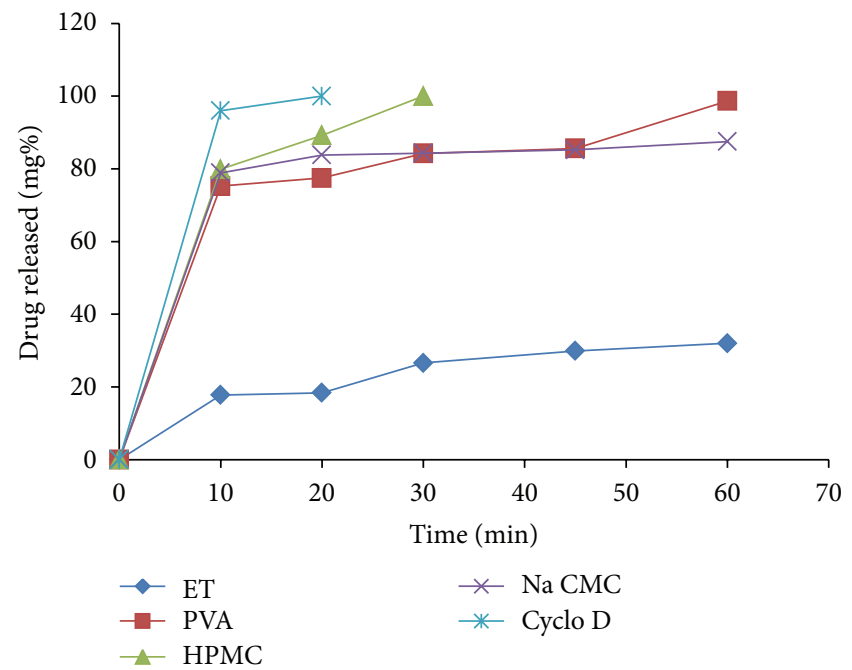

(b)

FIGURE 3: (a) Dissolution profiles of ET nanosuspensions prepared by the $\mathrm{pH}$ shift method in distilled water. $P=0.03$ significant one-way ANOVA. (b) Dissolution profiles of ET nanosuspensions prepared by the $\mathrm{pH}$ shift method in distilled water. $P=0.024$ significant one way ANOVA.

of drug release after $10 \mathrm{~min} .\left(\mathrm{DR}_{10 \mathrm{~min}}\right)$ and percentage dissolution efficiency at $10 \mathrm{~min}\left(\mathrm{DE}_{10 \mathrm{~min}}\right)$. The \% $\mathrm{DE}$ of the drug dissolved after $10 \mathrm{~min}$ of dissolution experiment was used as a parameter for comparison between the pure drug and nanosuspension formulations (Table 3). ET is a weak organic acid, with pKa of 4.5 and its solubility increases with increasing the $\mathrm{pH}$. It is poorly water soluble drug therefore, water and $\mathrm{pH} 6.8$ were selected as dissolution media to slow and enhance dissolution rate of ET, respectively, and as a result allow greater discrimination of processing efficacy.

The dissolution profiles of lyophilized ET nano-solid suspensions prepared by the $\mathrm{pH}$ shift method, in comparison with crude drug as a reference are shown in Figures 3 and 4. The dissolution rate of ET in distilled water was markedly enhanced in the nanosolid suspension, as more than $65 \%$ of drug dissolved in $10 \mathrm{~min}$ from all the nanosuspension formulations except F5 and F8, as compared to less than $20 \%$ of crude drug. This could be attributed to the increase in the surface area of the drug and possibly better wettability and dispersion of nanosolid suspensions in dissolution medium. These results were emphasized by $\% \mathrm{DE}_{10 \mathrm{~min}}$ after $10 \mathrm{~min}$ as shown in Table 3 as F5 and F8 exhibited the lowest DE.

It was reported that the saturation solubility increases with decreasing particle size. However, this effect is only pronounced for particle below approximately $2 \mu \mathrm{m}$, especially below $1 \mu \mathrm{m}[53]$.

$\beta$-Cyclodextrin containing nanosuspension with particle size of $866 \mathrm{~nm}$ showed the highest drug dissolution rate 


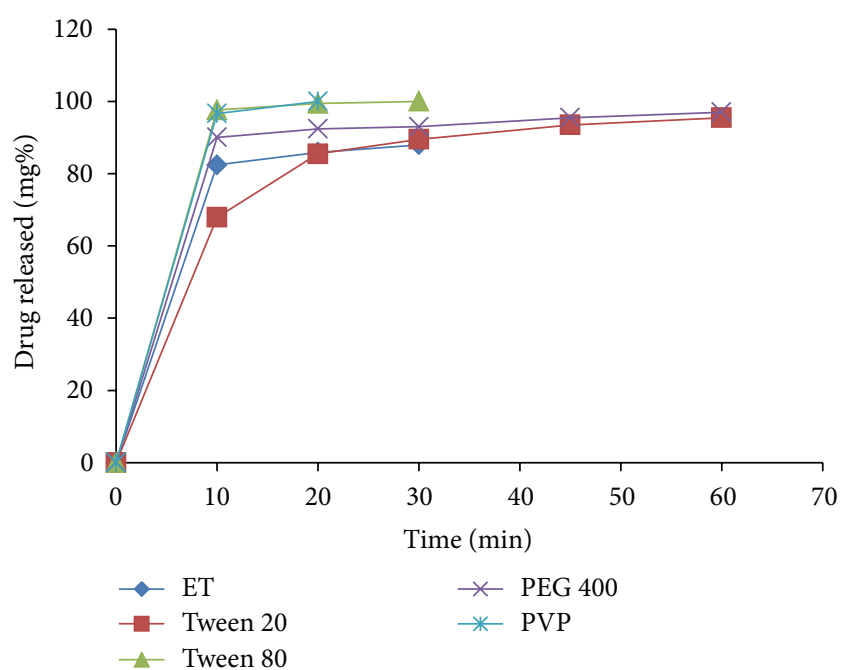

(a)

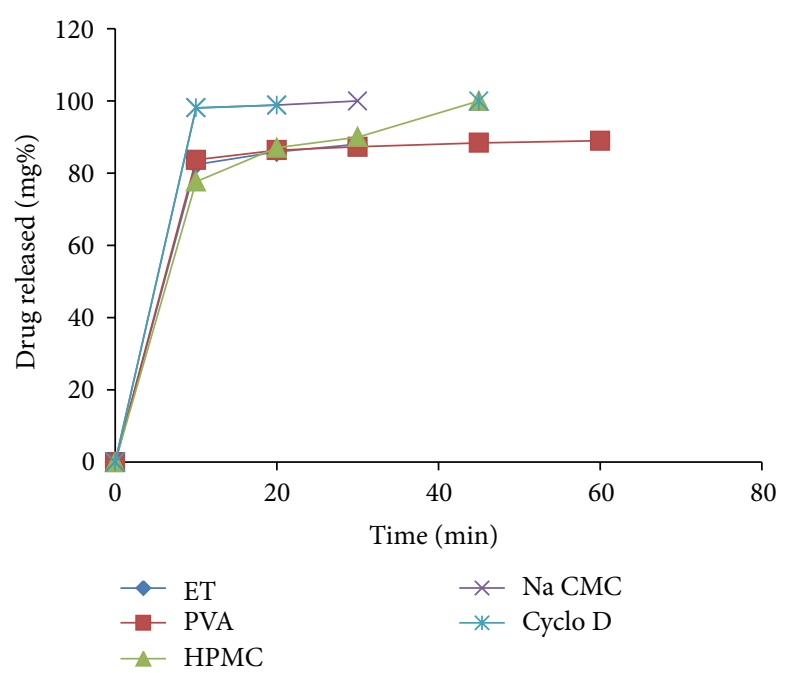

(b)

FIGURE 4: (a) Dissolution profiles of ET nanosuspensions prepared by $\mathrm{pH}$ shift method in phosphate buffer $\mathrm{pH}$ 6.8. $\mathrm{P}=0.96$ not significant one-way ANOVA but $P=0.01$ significant two-way ANOVA. (b) Dissolution profiles of ET nanosuspensions prepared by $\mathrm{pH}$ shift method in phosphate buffer $\mathrm{pH}$ 6.8. $P=0.97$ not significant one-way ANOVA, $P=0.0011$ significant two-way ANOVA.

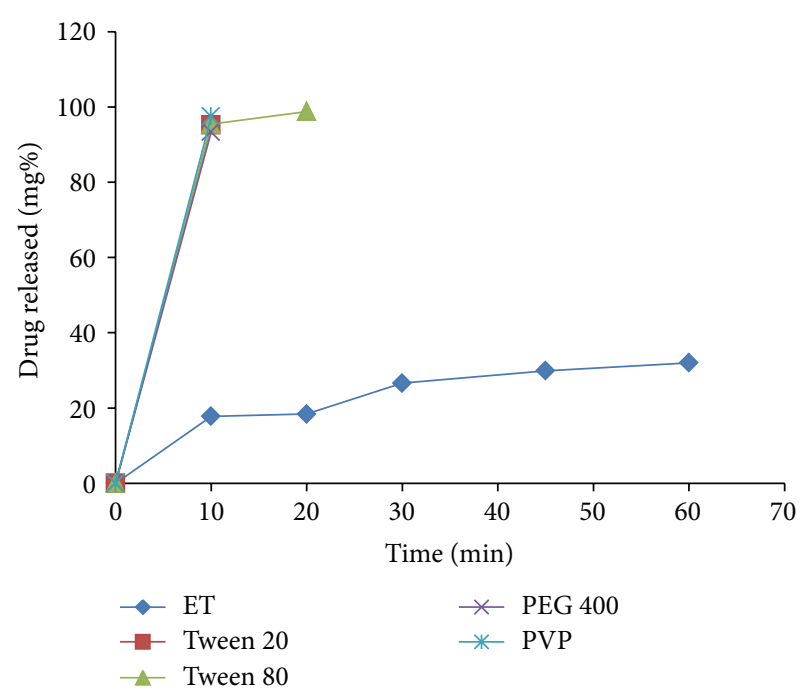

(a)

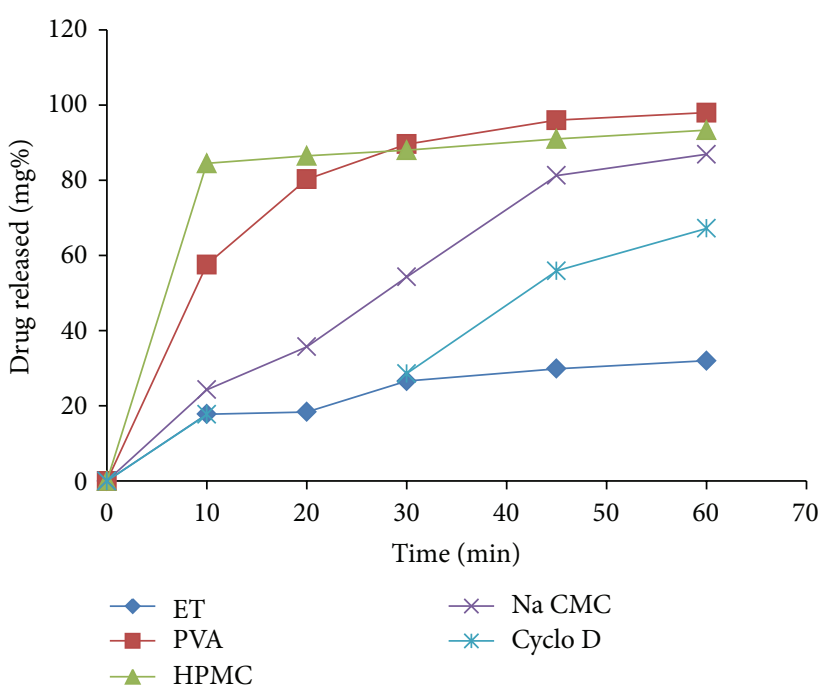

(b)

Figure 5: (a) Dissolution profiles of ET nanosuspensions prepared by antisolvent method in distilled water. $P=0.024$ significant one-way ANOVA. (b) Dissolution profiles of ET nanosuspensions prepared by antisolvent method in distilled water. $P=0.018$ significant one-way ANOVA.

as $96 \%$ of drug dissolved in 10 minutes whereas, HPMC containing nanosuspension with particle size of $1025 \mathrm{~nm}$ showed about $79.9 \%$ of drug dissolved within 10 minute of dissolution test. The reduction in particle size from $1025 \mathrm{~nm}$ in case of HPMC to $866 \mathrm{~nm}$ in case of $\beta$-Cyclodextrin showed a significant increase in drug dissolution rate $(P<0.05)$. Tween 80 containing nanosuspension with particle size of $393 \mathrm{~nm}$ resulted in about $77.65 \%$ of drug dissolved in 10 minute. Further decrease in particle size from $866 \mathrm{~nm}$ in case of $\beta$-Cyclodextrin containing nanosuspension to $393 \mathrm{~nm}$ in
Tween 80 containing nanosuspension resulted in a decrease in drug dissolution rate. It was found that the $\%$ drug dissolved decreased significantly from $96 \%$ (in case of $\beta$ Cyclodextrin) to $77.65 \%$ (in case of Tween 80$)(P<0.05)$. It can be seen that the predominant factor in drug dissolution was not the particle size but the mechanism by which the stabilizer used increase drug dissolution.

The increase in the dissolution rate of ET from $\beta$-Cyclodextrin containing nanosuspension may be due to the formation of water-soluble inclusion complexes with $\beta$-CD. 


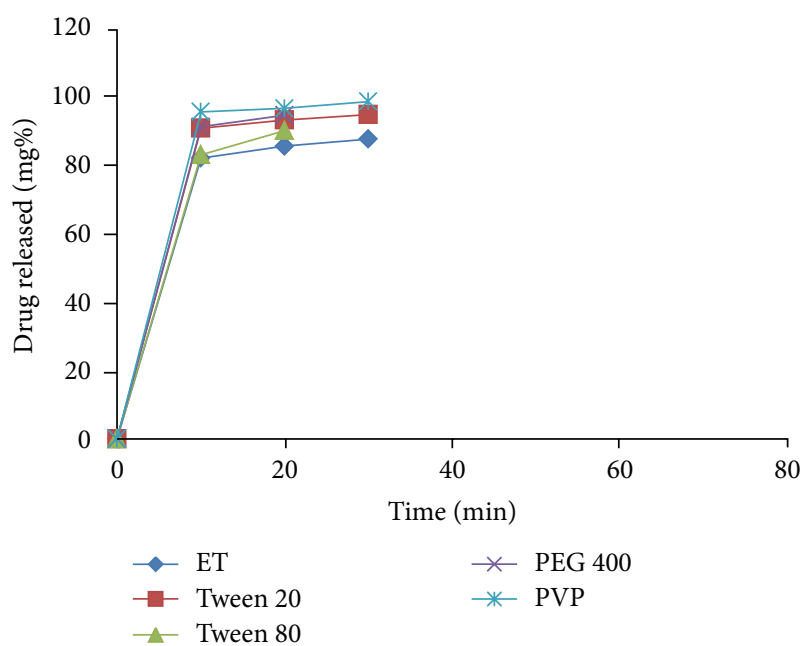

(a)

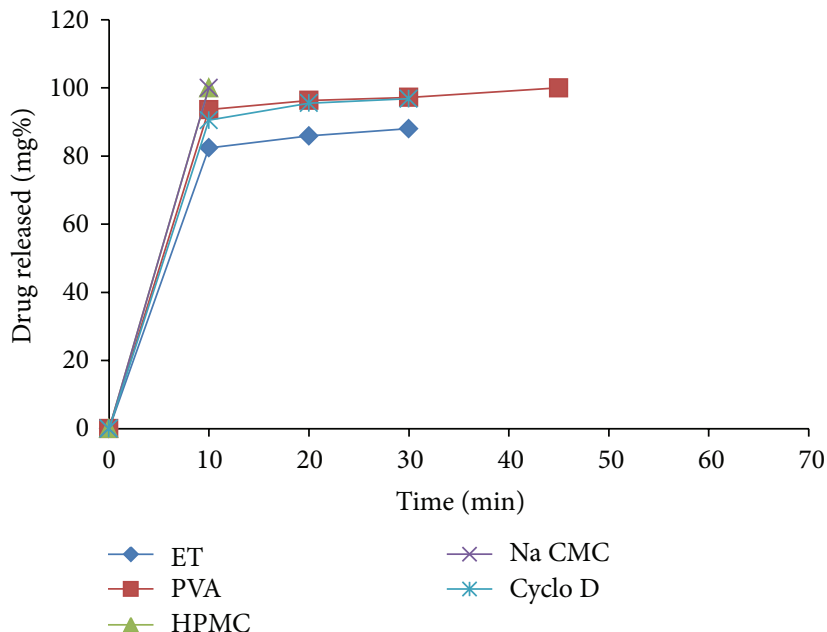

(b)

FIGURE 6: (a) Dissolution profiles of ET nanosuspensions prepared by antisolvent method in phosphate buffer $\mathrm{pH}$ 6.8. $P=0.993$ not significant one-way ANOVA $P=0.001$ significant two-way ANOVA. (b) Dissolution profiles of ET nanosuspensions prepared by antisolvent method in phosphate buffer $\mathrm{pH}$ 6.8. Not significant one way ANOVA but significant two-way ANOVA $P<0.001$.

The drug molecule is associated with $\beta$-CD by noncovalent and weak intermolecular forces. The interactions between the hydrophobic part of drug molecule and the polar cavity causes dehydration of the hydrophobic drug molecule, thereby increasing the affinity toward water and hence increasing the dissolution [54]. The surfactant-like properties of CDs can also be postulated to explain the higher dissolution rate of the complexes [55]. CDs can also reduce the interfacial tension between the solid particles of the drug and the dissolution medium, leading to a greater rate of dissolution [56].

The percentage of drug dissolved ranged from 96 ( $\beta$ Cyclodextrin) to 39 (PVP) which seemed to be affected by relative viscosity of the polymeric dispersion at the vicinity of the particle surface. Furthermore, the dissolution rate is affected by the wetting ability of the stabilizers used which followed the trend: $(\beta$-Cyclodextrin) $>$ HPMC $>$ PEG 400 $>\mathrm{Na} \mathrm{CMC}>$ Tween $80>$ PVA $>$ Tween $20>$ PVP. Table 3 showed that the nanosuspension containing $\beta$-Cyclodextrin as stabilizer exhibited the highest $\mathrm{DE}_{10 \text { min }}(48 \%)$, whereas nanosuspension of PVP showed the lowest $\mathrm{DE}_{10 \text { min }}(7.56 \%)$.

Using phosphate buffer pH 6.8 as dissolution medium, the crude drug showed a significant increase in its dissolution rate under sink condition. Dissolution rate study of the drug in distilled water showed that about $17.8 \%$ of drug dissolved in water within 10 minutes. Changing the dissolution medium to buffer $\mathrm{pH} 6.8$ the percentage of dissolved drug increased to 82.4 at the same time $(P<0.05)$.

Nanosuspensions of $\beta$-Cyclodextrin, Na CMC and Tween 80 showed the highest drug dissolution rate as they released about $98 \%$ of their drug content within 10 minutes, although their particle size are $866,827 \mathrm{~nm}$ and 393 , respectively. The obtained results may attributed to the fast dissolution of the drug in $\mathrm{pH} 6.8$ thus facilitates its release from the host $\beta$ Cyclodextrin structure. $\mathrm{Na} C M C$ dissolves better in buffer $\mathrm{pH}$
6.8; thus it increased the wettability and dissolution of ET. In case of Tween 80 containing nanosuspension; two factors may be considered; which are the small particle size and the good wettability of the drug particles. PVP, PVA, PEG and HPMC as stabilizers showed slower dissolution rate than the above mentioned stabilizers which may be to the viscosity imparted to the dissolution medium by the hydration and dissolution of these polymers in $\mathrm{pH}$ 6.8. The efficacy of the different stabilizers on drug dissolution rate are in the following order; $\beta$-Cyclodextrin $=\mathrm{Na} \mathrm{CMC}=$ Tween $80>$ PVP $>$ PEG $>$ PVA $>$ HPMC $>$ Tween 20. The $\% \mathrm{DE}_{10 \text { min }}$ of PVP nanosuspension formulation was 46.33 , whereas it was 11.85 for Tween 20 .

Dealing with the antisolvent method Figures 5 and 6; It was observed that PVP containing nanosuspension (p.s. of $290 \mathrm{~nm}$ ) exhibited the highest dissolution rate in distilled water as about $97.5 \%$ of drug dissolved in 10 minutes whereas, $\beta$-cyclodextrin containing formulation (p.s. of $517 \mathrm{~nm}$ ) showed the least drug dissolution rate as about $17.7 \%$ of drug dissolved in 10 minutes. Thus, the decrease in particle size from $517 \mathrm{~nm}$ in case of $\beta$-cyclodextrin to $290 \mathrm{~nm}$ in case of PVP showed a significant increase in the dissolution rate $(P<0.05)$. The effect of stabilizer used on dissolution rate of different formulations can be arranged in the following order; PVP $>$ Tween $20=$ Tween $80>$ PEG $>$ HPMC $>$ PVA $>$ $\mathrm{Na} \mathrm{CMC}>\beta$-cyclodextrin. The obtained results may be due predominantly to the nature of the stabilizer. The values of $\%$ $\mathrm{DE}_{10 \text { min }}$ are consistent with the previous order as DE of PVP nanosuspension was $49.75 \%$ and the lowest $\% \mathrm{DE}_{10}$ min was of $\beta$-cyclodextrin nanosuspension (4.39).

On the other hand, the effect of stabilizer used on dissolution rate in buffer $\mathrm{pH} 6.8$ of different formulations can be arranged in the following order; $\mathrm{Na} C M C=\mathrm{HPMC}>\mathrm{PVP}$ $>$ PVA $>$ PEG $=$ Tween $20>\beta$-cyclodextrin $>$ Tween 80 . The 


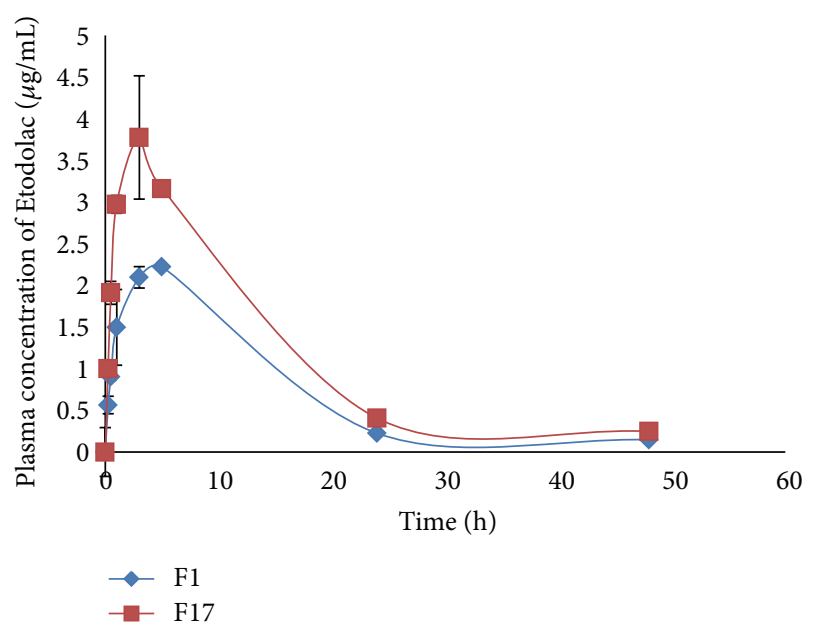

FIGURE 7: Mean plasma concentration-time profiles for F1 and F17 formulations after oral administration of a single dose of $20 \mathrm{mg} / \mathrm{kg}$. Each data point is the mean \pm SD from six experiments $(n=6)$.

$\mathrm{DE}_{10 \text { min }}$ value of both $\mathrm{Na}$ CMC and HPMC nanosuspensions was $49.5 \%$ as shown in Table 3.

Since nanoparticles prepared by antisolvent method using Tween 80 as a stabilizer showed a promising results concerning the particle size and dissolution rate, it was selected for further in vivo study.

3.8. In Vivo Performance. In the present investigation, the pharmacokinetic parameters of a nanosuspension of ET (F17) were compared to that of pure ET suspension (F1) after administration by oral gastric catheter in rats. Figure 7 shows the mean plasma ET concentration-time curves of the two formulations. The pharmacokinetic parameters are summarized in Table 2. Nanosuspension of ET showed higher average plasma concentration than the pure drug suspension formulation. Thus, the preparation of ET in the form of nanosuspension improved its bioavailability. This result was also consistent with the dissolution profile that the dissolution rate of ET (F17) was faster than that of pure ET in both water and $\mathrm{pH} 6.8$ as dissolution media. The peak plasma concentration $\left(C_{\max }\right)$ for pure ET suspension $(\mathrm{F} 1)$ was found to be $2.38 \mu \mathrm{g} \cdot \mathrm{mL}^{-1}$ and the time to attain peak concentration $\left(T_{\max }\right)$ was $4 \mathrm{~h}$, whereas, peak plasma concentration for ET nanosuspension (F17) was $3.78 \mu \mathrm{g} \cdot \mathrm{mL}^{-1}$ which was attained in $3 \mathrm{~h}$. This result indicated the faster absorption of ET from the nanosuspension (F17). Pure ET suspension did not show significantly lower plasma level up to the first $1 \mathrm{~h}$ when compared with ET nanosuspension ( $P=0.0555$, considered not quite significant). But it showed significantly lower plasma level up to the $3 \mathrm{hr}$ compared to F17 $(P=0.018$, considered significant). At 5-48 h, Pure ET suspension did not show significantly lower plasma level compared to F17 $(P=0.0676$, considered not quite significant). These results indicated that the two formulations showed similar elimination profile (Table 4). Etodolac nanosuspension (F17) showed significantly larger $\mathrm{AUC}_{0-48}$ compared to pure ET suspension (F1) ( $P=0.0034$, considered significant).
TABLE 4: Pharmacokinetic parameters after oral administration of ET formulations.

\begin{tabular}{lcc}
\hline $\begin{array}{l}\text { Pharmacokinetic } \\
\text { parameter }\end{array}$ & $\begin{array}{c}\text { Formulation A } \\
(\text { Mean } \pm \mathrm{SD})^{*}\end{array}$ & $\begin{array}{c}\text { Formulation B } \\
(\text { Mean } \pm \mathrm{SD})^{*}\end{array}$ \\
\hline $\mathrm{AUC}_{0-48}\left(\mu \mathrm{g} \cdot \mathrm{h} \cdot \mathrm{mL}^{-1}\right)$ & $36.5 \pm 2.27$ & $55.07 \pm 9.88(63.417)$ \\
$\mathrm{AUC}_{0-\infty}\left(\mu \mathrm{g} \cdot \mathrm{h} \cdot \mathrm{mL}^{-1}\right)$ & $38.16 \pm 2.26$ & $58.20 \pm 9.55$ \\
$C_{\max } \mu \mathrm{g} \cdot \mathrm{mL}^{-1}$ & $2.38 \pm 0.30$ & $3.78 \pm 0.13$ \\
$T_{\max }(\mathrm{h})$ & 4.0 & 3.0 \\
$t_{1 / 2}(\mathrm{~h})$ & $10.93 \pm 0.62$ & $11.60 \pm 0.97$ \\
$K_{\mathrm{el}}(\mathrm{h})$ & $0.063 \pm 0.004$ & $0.06 \pm 0.01$ \\
$\mathrm{MRT}(\mathrm{h})$ & $11.51 \pm 0.77$ & $12.92 \pm 0.27$ \\
$\%$ absorbed in $1 \mathrm{~h}$ & $7.79 \pm 0.26$ & $21.20 \pm 0.69$ \\
Clearance rate & 132 & 86 \\
$(\mathrm{mg} / \mathrm{mL})$ & &
\end{tabular}
${ }^{*} n=6$.

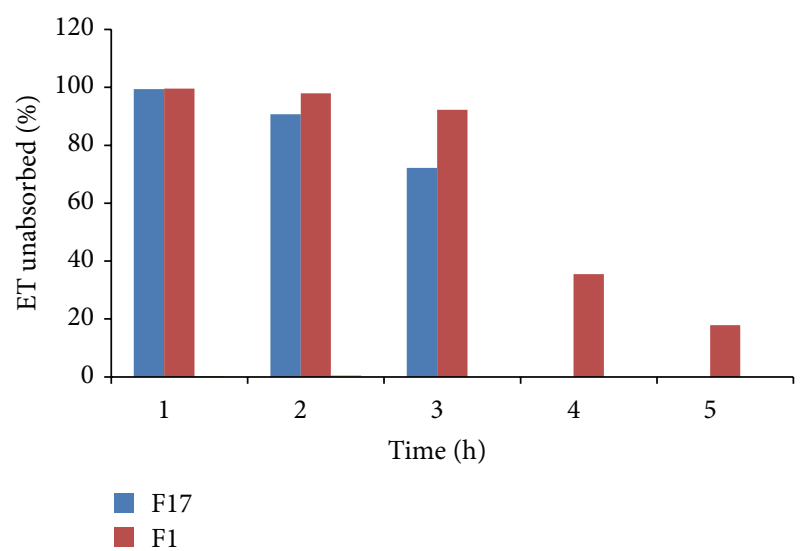

FIGURE 8: Percentage ET unabsorbed versus time for the two formulations F1 (Pure ET suspension) and F17 (ET nanosuspension).

In order to demonstrate a faster absorption of ET nanosuspension (F17), particularly during the first hour after administration, the percentage of ET absorbed after $1 \mathrm{~h}$ was estimated (Table 4). The percentage absorbed from F1 and F17 was 7.79 and 21.20, respectively. Nanoparticles of ET were well absorbed, with an percentage absorption value 2.7 times more than that of micrometric size of ET. As can be expected, the clearance rate of nanoparticles ET was lower $\left(86.20 \mathrm{mg} \cdot \mathrm{mL}^{-1}\right)$ than ET microparticles $\left(132 \mathrm{mg} \cdot \mathrm{mL}^{-1}\right)$ because clearance is more readily estimated by dividing dose by AUC. Therefore, the elimination half-life $\left(t_{1 / 2}\right)$ of F17 is slightly higher (11.6 h) than that of F1 (10.9).

Figure 8 represents a plot of the percentage of unabsorbed ET versus time after oral administration of the two formulations. A linear relationship can be observed between logarithm unabsorbed values and time with $R^{2}$ equal to 0.994 and 0.997 for F1 and F17, respectively, which suggests apparent first-order absorption [57]. The apparent first-order absorption rate constants $\left(K_{\mathrm{ab}}\right)$ were estimated from the slope of the curves multiplied by 2.303 , and were found to be $0.274 \pm$ 0.29 and $1.679 \pm 0.92 \mathrm{hr}^{-1}$ for F1 and F17, respectively. These results indicate the faster absorption rate of ET from F17 
compared to that from F1. Thus These results supported the in vitro dissolution data, where the dissolution was significantly increased in F17 compared to F1.

The improved oral bioavailability of ET could be explained by the combination of the following effects: firstly, the drug molecules were absorbed rapidly from gastrointestinal wall due to the significantly improved dissolution rate by the reduced particle size with increased surface area and reduced diffusion layer thickness [13]. Secondly, An increase in adhesion surface area between nanoparticles and the mucin layer coating intestinal epithelium of villi facilitates the nanosized drug to traverse the mucin layer and the epithelial cells, resulting in an increase in oral absorption of the nanosized drug; compared with large particles, nanoparticles in general possess a stronger curvature of the surface, which produces more dissolution pressure with a corresponding increase in saturation solubility $[19,58]$. The increase in saturation solubility, in turn, favors increased concentration gradient between intestinal epithelial cells and the mesenteric circulation beneath; and an increased dissolution velocity of the drug, which overcomes this rate-limiting step in the drug absorption process. In addition, the diffusion distance on the surface of drug nanoparticles is decreased, causing an increased concentration gradient [59]. An increase in surface area and concentration gradient leads to a more pronounced increase in the dissolution rate compared with a micronized product. Saturation solubility and dissolution rate are important parameters affecting the bioavailability of orally administered drugs. Drug nanosization can reduce erratic drug absorption so the adhesion process of drug nanoparticles to mucosal surface would be highly reproducible and not very affected by the nutritional and food status of patients. It has been reported that smaller particles of drugs are taken up more easily by macrophages and obtain a higher deposition rate, and hence a better therapeutic index [60]. Based on these favorable characteristics, nanosization has the potential to overcome absorption limitations of poorly soluble drugs.

\section{Conclusion}

ET nanosuspensions were prepared by $\mathrm{pH}$ shift or antisolvent method in presence of different stabilizers with drug to stabilizer ratio of $3: 1$. Solvent precipitation method succeeded in producing particles in the nanosized range. The dissolution of nanosized ET was significantly enhanced compared with the crude pure drug. The results showed that the particle size minimization produced by $\mathrm{pH}$ shift method was not the major determining factor in the dissolution improvement. Rather, the type of stabilizer used in the formulations was of greater importance. The results also demonstrated that nanoprecipitation can thus be a simple and effective approach to produce submicron particles of poorly water-soluble drugs. The in vivo test demonstrated that the $C_{\max }$ and $\mathrm{AUC}_{0-48}$ values of nanosuspension were approximately 1.6 and 1.5 fold, respectively, greater than that of the crude drug $(P<0.05)$. Nanoparticles of ET nanosuspensions were well absorbed, with a percentage drug absorption value 2.7 times more than that of micrometric size of ET. Nanosnization of Etodolac had the potential to overcome absorption limitations of the poorly soluble drug.

\section{Conflict of Interests}

The authors declare that there is no conflict of interests regarding the publication of this paper.

\section{Acknowledgment}

This research project was supported by a grant from the "Research Center of the Female Scientific and Medical Colleges," Deanship of Scientific Research, King Saud University.

\section{References}

[1] E. Merisko-Liversidge, G. G. Liversidge, and E. R. Cooper, "Nanosizing: a formulation approach for poorly-water-soluble compounds," European Journal of Pharmaceutical Sciences, vol. 18, no. 2, pp. 113-120, 2003.

[2] R. H. Müller and K. Peters, "Nanosuspensions for the formulation of poorly soluble drugs. I. Preparation by a size-reduction technique," International Journal of Pharmaceutics, vol. 160, no. 2, pp. 229-237, 1998.

[3] K. R. Vandana, Y. Prasanna Raju, V. Harini Chowdary, M. Sushma, and N. Vijay Kumar, "An overview on in situ micronization technique-an emerging novel concept in advanced drug delivery," Saudi Pharmaceutical Journal, vol. 22, no. 4, pp. 283-289, 2014.

[4] A. K. Nayak and P. P. Panigrahi, "Solubility enhancement of etoricoxib by cosolvency approach," ISRN Physical Chemistry, vol. 2012, Article ID 820653, 5 pages, 2012.

[5] S. Sinha, M. Ali, S. Baboota, A. Ahuja, A. Kumar, and J. Ali, "Solid dispersion as an approach for bioavailability enhancement of poorly water-soluble drug ritonavir," AAPS PharmSciTech, vol. 11, no. 2, pp. 518-527, 2010.

[6] K. T. Savjani, A. K. Gajjar, and J. K. Savjani, "Drug solubility: importance and enhancement techniques," ISRN Pharmaceutics, vol. 2012, Article ID 195727, 10 pages, 2012.

[7] C. Jacobs, O. Kayser, and R. H. Müller, "Nanosuspensions as a new approach for the formulation for the poorly soluble drug tarazepide," International Journal of Pharmaceutics, vol. 196, no. 2, pp. 161-164, 2000.

[8] P. Kocbek, S. Baumgartner, and J. Kristl, "Preparation and evaluation of nanosuspensions for enhancing the dissolution of poorly soluble drugs," International Journal of Pharmaceutics, vol. 312, no. 1-2, pp. 179-186, 2006.

[9] L. Gao, G. Liu, J. Ma, X. Wang, L. Zhou, and X. Li, "Drug nanocrystals: in vivo performances," Journal of Controlled Release, vol. 160, no. 3, pp. 418-430, 2012.

[10] M. Yadav, S. Dhole, and P. Chavan, "Nanosuspension: a novel techniques in drug delivery system," World Journal of Pharmacy and Pharmaceutical Sciences, vol. 3, no. 2, pp. 410-433, 2014.

[11] B. H. L. Böhm and R. H. Müller, "Lab-scale production unit design for nanosuspensions of sparingly soluble cytotoxic drugs," Pharmaceutical Science and Technology Today, vol. 2, no. 8, pp. 336-339, 1999.

[12] V. B. Patravale, A. A. Date, and R. M. Kulkarni, "Nanosuspensions: a promising drug delivery strategy," Journal of Pharmacy and Pharmacology, vol. 56, no. 7, pp. 827-840, 2004. 
[13] R. J. Hintz and K. C. Johnson, "The effect of particle size distribution on dissolution rate and oral absorption," International Journal of Pharmaceutics, vol. 51, no. 1, pp. 9-17, 1989.

[14] B. E. Rabinow, "Nanosuspensions in drug delivery," Nature Reviews Drug Discovery, vol. 3, no. 9, pp. 785-796, 2004.

[15] K. Peters, S. Leitzke, J. E. Diederichs et al., "Preparation of a clofazimine nanosuspension for intravenous use and evaluation of its therapeutic efficacy in murine Mycobacterium avium infection," Journal of Antimicrobial Chemotherapy, vol. 45, no. 1, pp. 77-83, 2000.

[16] M. J. Grau, O. Kayser, and R. H. Müller, "Nanosuspensions of poorly soluble drugs-reproducibility of small scale production," International Journal of Pharmaceutics, vol. 196, no. 2, pp. 155-159, 2000.

[17] J. Chingunpituk, "Nanosuspension technology for drug delivery," Walailak Journal of Science and Technology, vol. 4, no. 2, pp. 139-153, 2007.

[18] X. Pu, J. Sun, M. Li, and Z. He, "Formulation of nanosuspensions as a new approach for the delivery of poorly soluble drugs," Current Nanoscience, vol. 5, no. 4, pp. 417-427, 2009.

[19] P. Lakshmi and G. A. Kumar, "Nanosuspension technology: a review," International Journal of Pharmacy and Pharmaceutical Sciences, vol. 2, no. 4, pp. 35-40, 2010.

[20] G. G. Liversidge and P. Conzentino, "Drug particle size reduction for decreasing gastric irritancy and enhancing absorption of naproxen in rats," International Journal of Pharmaceutics, vol. 125, no. 2, pp. 309-313, 1995.

[21] A. Papdiwal, V. Pande, and K. Sagar, "Design and characterization of zaltoprofen nanosuspension by precipitation method," Der Pharma Chemica, vol. 6, no. 3, p. 161, 2014.

[22] M. Kakran, N. G. Sahoo, I.-L. Tan, and L. Li, "Preparation of nanoparticles of poorly water-soluble antioxidant curcumin by antisolvent precipitation methods," Journal of Nanoparticle Research, vol. 14, no. 3, article 757, 2012.

[23] D. Xia, P. Quan, H. Piao, S. Sun, Y. Yin, and F. Cui, "Preparation of stable nitrendipine nanosuspensions using the precipitationultrasonication method for enhancement of dissolution and oral bioavailability," European Journal of Pharmaceutical Sciences, vol. 40, no. 4, pp. 325-334, 2010.

[24] B. P. Sahu and M. K. Das, "Formulation, optimization, and in vitro/in vivo evaluation of furosemide nanosuspension for enhancement of its oral bioavailability," Journal of Nanoparticle Research, vol. 16, no. 4, article 2360, 2014.

[25] M. R. Bhalekar, P. G. Upadhaya, S. Reddy, S. J. Kshirsagar, and A. R. Madgulkar, "Formulation and evaluation of acyclovir nanosuspension for enhancement of oral bioavailability," Asian Journal of Pharmaceutics, vol. 8, no. 2, p. 110, 2014.

[26] A. Rezaei Mokarram, A. Kebriaee zadeh, M. Keshavarz, A. Ahmadi, and B. Mohtat, "Preparation and in-vitro evaluation of indomethacin nanoparticles," DARU, vol. 18, no. 3, pp. 185-192, 2010.

[27] J. A. Balfour and M. M.-T. Buckley, "Etodolac. A reappraisal of its pharmacology and therapeutic use in rheumatoid diseases and pain states," Drugs, vol. 42, no. 2, pp. 274-299, 1991.

[28] R. Talari, J. Varshosaz, S. A. Mostafavi, and A. Nokhodchi, "Gliclazide microcrystals prepared by two methods of in situ micronization: pharmacokinetic studies in diabetic and normal rats," AAPS PharmSciTech, vol. 11, no. 2, pp. 786-792, 2010.

[29] W. Martindale, Martindale: The Extra Pharmacopoeia, Rittenhouse Book Distributors, 1996.
[30] A. Maccagno, E. di Giorgio, and A. Romanowicz, "Effectiveness of etodolac ('Lodine') compared with naproxen in patients with acute gout," Current Medical Research and Opinion, vol. 12, no. 7, pp. 423-429, 1991.

[31] J. F. Mullane, "Etodolac for treatment of gout," Google Patents, 1987.

[32] A. Okamoto, T. Shirakawa, T. Bito et al., "Etodolac, a selective cyclooxygenase-2 inhibitor, induces upregulation of E-cadherin and has antitumor effect on human bladder cancer cells In Vitro and In Vivo," Urology, vol. 71, no. 1, pp. 156-160, 2008.

[33] N. Tsuneoka, Y. Tajima, A. Kitazato et al., "Chemopreventative effect of a cyclooxygenase-2-specific inhibitor (etodolac) on chemically induced biliary carcinogenesis in hamsters," Carcinogenesis, vol. 26, no. 2, pp. 465-469, 2005.

[34] K. Glaser, "Cyclooxygenase selectivity and NSAIDs: cyclooxygenase-2 selectivity of etodolac (Lodine)," in Side Effects of Anti-Inflammatory Drugs IV, pp. 211-221, Springer, Berlin, Germany, 1997.

[35] J.-M. Shi, S.-G. Lai, C.-J. Xu, G.-L. Duan, and D. Li, "Pharmacokinetic difference between S-(+)- and R-(-)-etodolac in rats," Acta Pharmacologica Sinica, vol. 25, no. 8, pp. 996-999, 2004.

[36] L. X. Yu, G. L. Amidon, J. E. Polli et al., "Biopharmaceutics classification system: the scientific basis for biowaiver extensions," Pharmaceutical Research, vol. 19, no. 7, pp. 921-925, 2002.

[37] Y. Naito, H. Matsuda, K. Shimomura, K. Kurihara, K. Tochigi, and K. Tomono, "Measurement and correlation of solubilities of the poorly water-soluble pharmaceutical compound etodolac by addition of co-solvents," Fluid Phase Equilibria, vol. 357, no. 2, pp. 43-49, 2013.

[38] L. S. Taylor and G. Zografi, "Spectroscopic characterization of interactions between PVP and indomethacin in amorphous molecular dispersions," Pharmaceutical Research, vol. 14, no. 12, pp. 1691-1698, 1997.

[39] B. Gorain, H. Choudhury, U. Nandi, A. Das, S. Dan, and T. K. Pal, "Development and validation of an HPLC method for simultaneous detection and quantification of paracetamol and etodolac in human plasma and its application to a pharmacokinetic study," Journal of AOAC International, vol. 96, no. 3, pp. 573-579, 2013.

[40] J. G. Wagner and E. Nelson, "Kinetic analysis of blood levels and urinary excretion in the absorptive phase after single doses of drug," Journal of Pharmaceutical Sciences, vol. 53, no. 11, pp. 1392-1403, 1964.

[41] D. Perrier and M. Gibaldi, Pharmacokinetics, 2nd edition, 1982.

[42] B. Sanford and B. Charles, Pharmaceutical Statistics: Practical and Clinical Applications, 4th edition, 2004.

[43] S. Kumar and D. J. Burgess, "Nanosuspensions," in Long Acting Injections and Implants, pp. 239-261, Springer, 2012.

[44] Y. Dong, W. K. Ng, S. Shen, S. Kim, and R. B. H. Tan, "Preparation and characterization of spironolactone nanoparticles by antisolvent precipitation," International Journal of Pharmaceutics, vol. 375, no. 1-2, pp. 84-88, 2009.

[45] L. Peltonen and J. Hirvonen, "Pharmaceutical nanocrystals by nanomilling: critical process parameters, particle fracturing and stabilization methods," Journal of Pharmacy and Pharmacology, vol. 62, no. 11, pp. 1569-1579, 2010.

[46] P. R. Mishra, L. A. Shaal, R. H. Müller, and C. M. Keck, "Production and characterization of Hesperetin nanosuspensions for dermal delivery," International Journal of Pharmaceutics, vol. 371, no. 1-2, pp. 182-189, 2009. 
[47] D. J. Patel, J. K. Patel, and V. M. Pandya, "Improvement in the dissolution of poorly water soluble drug using media milling technique," Thai Journal of Pharmaceutical Sciences, vol. 34, no. 4, pp. 155-164, 2010.

[48] Z. Zhu, K. Margulis-Goshen, S. Magdassi, Y. Talmon, and C. W. Macosko, "Polyelectrolyte stabilized drug nanoparticles via flash nanoprecipitation: a model study with beta-carotene," Journal of Pharmaceutical Sciences, vol. 99, no. 10, pp. 42954306, 2010.

[49] E. Che, X. Zheng, C. Sun, D. Chang, T. Jiang, and S. Wang, "Drug nanocrystals: a state of the art formulation strategy for preparing the poorly water-soluble drugs," Asian Journal of Pharmaceutical Sciences, vol. 7, no. 2, pp. 85-95, 2012.

[50] Y. Chen, J. Liu, X. Yang, X. Zhao, and H. Xu, "Oleanolic acid nanosuspensions: preparation, in-vitro characterization and enhanced hepatoprotective effect," Journal of Pharmacy and Pharmacology, vol. 57, no. 2, pp. 259-264, 2005.

[51] B. P. Sahu and M. K. Das, "Preparation and in vitro/in vivo evaluation of felodipine nanosuspension," European Journal of Drug Metabolism and Pharmacokinetics, vol. 39, no. 3, pp. 183193, 2014.

[52] L. Wu, J. Zhang, and W. Watanabe, "Physical and chemical stability of drug nanoparticles," Advanced Drug Delivery Reviews, vol. 63, no. 6, pp. 456-469, 2011.

[53] J. Pardeike, D. M. Strohmeier, N. Schrödl et al., "Nanosuspensions as advanced printing ink for accurate dosing of poorly soluble drugs in personalized medicines," International Journal of Pharmaceutics, vol. 420, no. 1, pp. 93-100, 2011.

[54] S. Rawat and S. Jain, "Enhancement of intestinal absorption of few cox- 2 inhibitors through interaction with $\beta$-cyclodextrin," Indian Journal of Pharmaceutical Sciences, vol. 69, no. 4, pp. 529534, 2007.

[55] S.-Y. Lin and Y.-H. Kao, "Solid particulates of drug- $\beta$-cyclodextrin inclusion complexes directly prepared by a spray-drying technique," International Journal of Pharmaceutics, vol. 56, no. 3, pp. 249-259, 1989.

[56] M. Guyot, F. Fawaz, J. Bildet, F. Bonini, and A.-M. Lagueny, "Physicochemical characterization and dissolution of norfloxacin/cyclodextrin inclusion compounds and PEG solid dispersions,' International Journal of Pharmaceutics, vol. 123, no. 1, pp. 53-63, 1995.

[57] N. S. Barakat, "Enhanced oral bioavailability of etodolac by selfemulsifying systems: in-vitro and in-vivo evaluation," Journal of Pharmacy and Pharmacology, vol. 62, no. 2, pp. 173-180, 2010.

[58] C. Jacobs and R. H. Müller, "Production and characterization of a budesonide nanosuspension for pulmonary administration," Pharmaceutical Research, vol. 19, no. 2, pp. 189-194, 2002.

[59] M. Radtke, "Pure drug nanoparticles for the formulation of poorly soluble drugs," New Drugs, vol. 3, pp. 62-68, 2001.

[60] A. Lamprecht, U. Schäfer, and C.-M. Lehr, "Size-dependent bioadhesion of micro- and nanoparticulate carriers to the inflamed colonic mucosa," Pharmaceutical Research, vol. 18, no. 6, pp. 788-793, 2001. 

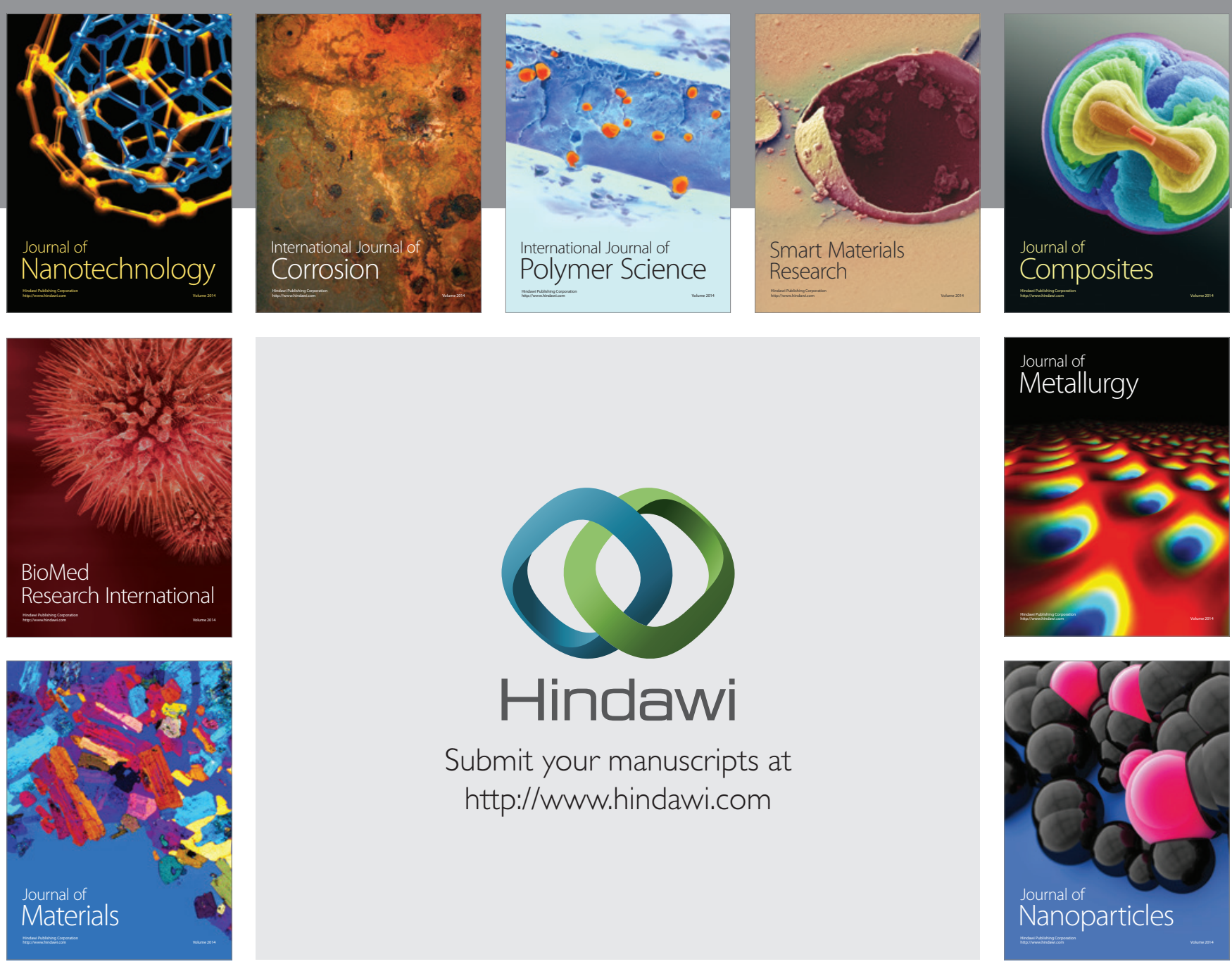

Submit your manuscripts at http://www.hindawi.com
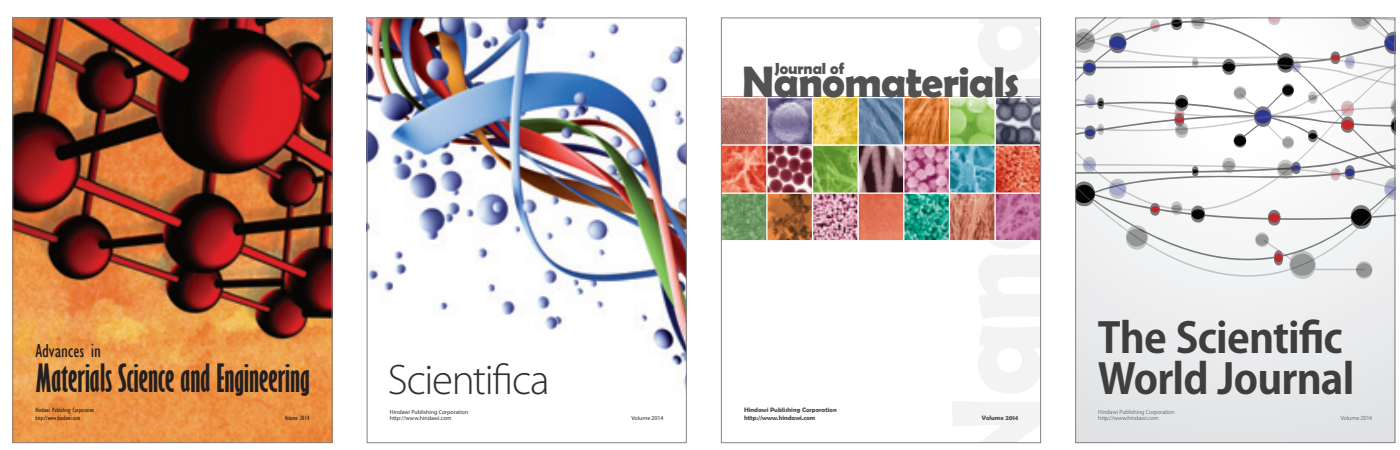

\section{The Scientific World Journal}
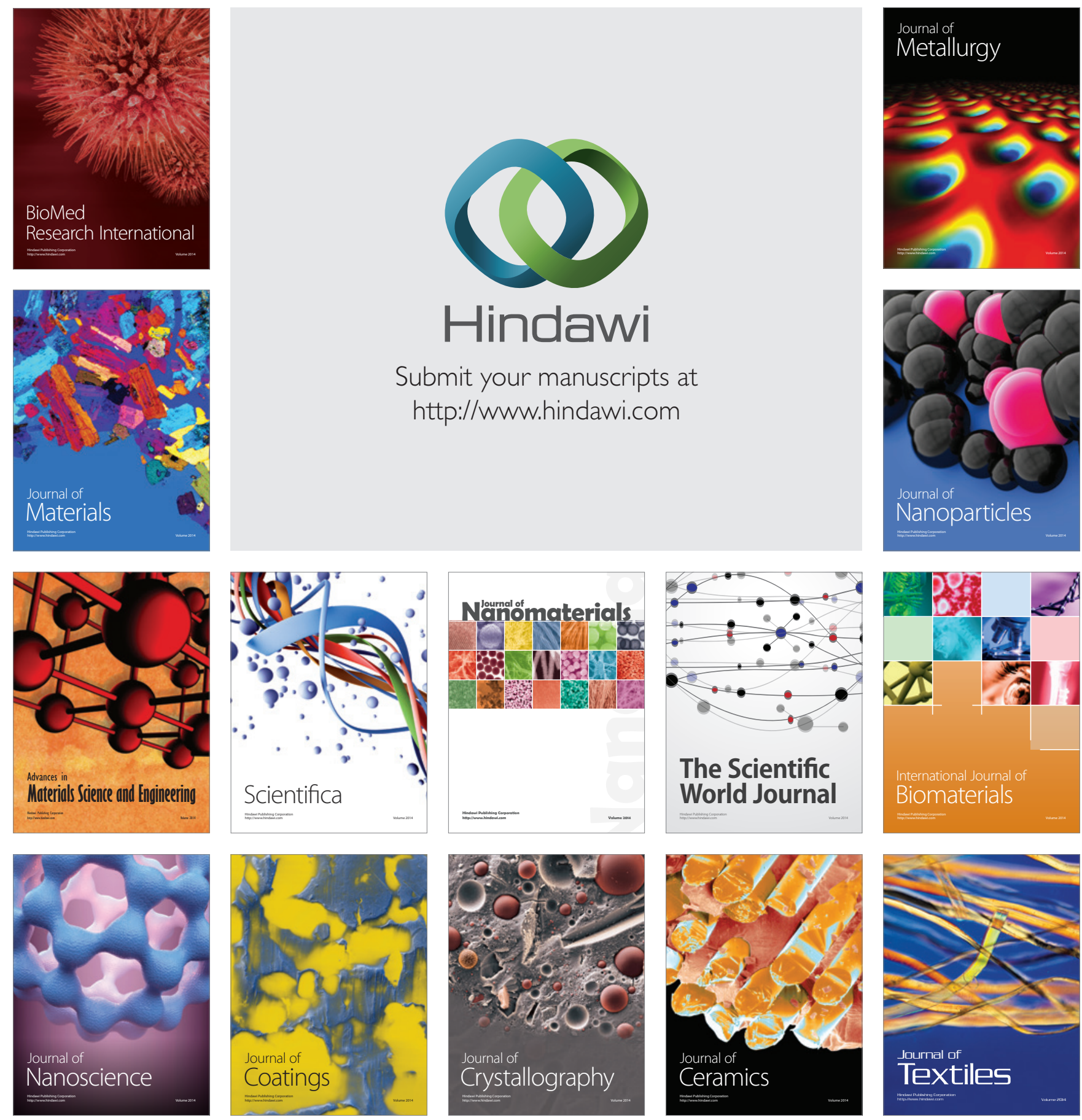